\title{
On the toughening of brittle materials by grain bridging: promoting intergranular fracture through grain angle, strength, and toughness
}

\author{
J.W. Foulk III ${ }^{\mathrm{a}}$,G.C. Johnson ${ }^{\mathrm{b}}$, P.A. Klein ${ }^{\mathrm{c}}$, R.O. Ritchie ${ }^{\mathrm{d}, \mathrm{e}, *}$ \\ a Sandia National Laboratory, Livermore, CA, 94550, USA \\ ${ }^{\mathrm{b}}$ Department of Mechanical Engineering, University of California, Berkeley, CA, \\ 94720, USA \\ ${ }^{\mathrm{c}}$ Franklin Templeton Investments, San Mateo, CA, 94403, USA \\ ${ }^{\mathrm{d}}$ Department of Materials Science and Engineering, University of California, \\ Berkeley, CA, 94720, USA \\ ${ }^{\mathrm{e}}$ Materials Sciences Division, Lawrence Berkeley National Laboratory, Berkeley, \\ CA, 94720, USA
}

\begin{abstract}
The structural reliability of many brittle materials such as structural ceramics relies on the occurrence of intergranular, as opposed to transgranular, fracture in order to induce toughening by grain bridging. For a constant grain boundary strength and grain boundary toughness, the current work examines the role of grain strength, grain toughness, and grain angle in promoting intergranular fracture in order to maintain such toughening. Previous studies have illustrated that an intergranular path and the consequent grain bridging process can be partitioned into five distinct regimes, namely: propagate, kink, arrest, stall and bridge. To determine the validity of the assumed intergranular path, the classical penentration/deflection problem of a crack impinging on an interface is reexamined within a cohesive zone framework for intergranular and transgranular fracture. Results considering both modes of propagation, i.e., a transgranular and intergranular path, reveal that crack-tip shielding is a natural outcome of the cohesive zone approach to fracture. Cohesive zone growth in one mode shields the opposing mode from the stresses required for cohesive zone initiation. Although stable propagation occurs when the required driving force is equivalent to the toughness for either transgranular or intergranular fracture, the mode of propagation depends on the normalized grain strength, normalized grain toughness, and grain angle. For each grain angle, the intersection of single path and multiple path solutions demarcates "strong" grains that increase the macroscopic toughness and "weak" grains that decrease it. The unstable transition to intergranular fracture reveals that an increasing grain toughness requires a growing region of the transgranular cohesive zone be at and near the peak cohesive strength. The
\end{abstract}

Preprint submitted to Journal of the Mechanics and Physics of Solids 30 November 2007 
inability of the body to provide the requisite stress field yields an overdriven and unstable configuration. The current results provide restrictions for the achievement of substantial toughening through intergranular fracture.

Key words: Fracture mechanisms, Fracture toughness, Ceramics, Finite elements, Crack bridging

\section{Introduction}

Modeling crack propagation at the microstructural level requires a thorough understanding of the driving force (Eshelby, 1951; Rice, 1968) coupled with the identification of the salient mechanisms aiding the resistance (e.g., Ritchie et al., 2000). It is often useful to partition the mechanisms of fracture into "intrinsic" and "extrinsic" processes. Intrinsic mechanisms evolve ahead of the crack tip (independent of crack size) while extrinsic mechanisms invariably evolve behind the crack tip (and dominate resistance-curve behavior). In nontransforming ceramics, grain and/or grain boundary fracture join a host of extrinsic mechanisms which may act in the crack wake (microcracking) and across the crack surfaces (grain bridging, grain sliding) to provide resistance to crack propagation. Experimental findings (Knehans and Steinbrech, 1982; Swanson et al., 1987; Gilbert et al., 1996; Becher et al., 1998) indicate that for most monolithic (non-transforming) structural ceramics, the most potent extrinsic mechanism is grain bridging.

Much of the research to date has focused on long-crack behavior at the "plateau" of the resistance curve (R-curve) as this invariably defines the highest toughness. Correspondingly less effort has been devoted to quantifying behavior for small crack extensions within the initials stages of the R-curve, although $\mathrm{Xu}$ et al. (1995) and Becher et al. (1996) did attempt to correlate microstructural parameters with a short-crack toughness. It is important to note here that for structural applications, the initial slope of the R-curve is in many respects more important than the peak (long-crack) toughness as it effectively governs the strength of the ceramic at realistically small crack sizes (Kruzic et al., 2005).

Prior work by the current authors (Foulk III et al., 2007) focused on predicting the evolution of grain bridging in this short-crack regime. To simplify matters, crack propagation was constrained to the grain boundaries (Zavattieri et al., 2001; Espinosa and Zavattieri, 2003a,b; Maiti et al., 2005). This work

\footnotetext{
* Corresponding author.

Email address: roritchie@lbl.gov (R.O. Ritchie).
} 
(Foulk III et al., 2007) showed that the grain bridging process, resulting from intergranular fracture of an inclined grain spanning two adjacent grains, can be generally partitioned into five regimes of resistance, namely crack propagation, kinking, arrest, stalling, and bridging with most toughening occurring during the stall regime and prior to actual bridge formation. These findings contrast previous analyses that did not consider bridge formation (Chantikul et al., 1990; Kovalev et al., 2000); moreover, they provides a feasible mechanism for the sharply rising R-curve behavior seen in many structural ceramics (Kruzic et al., 2005) and for the occurrence of crack reinitiation ahead of the stalled crack tip. ${ }^{*}$ Although the mechanics of crack reinitiation in lamellar solids differ from structural ceramics, the modeling efforts of Arata et al. (2001, 2002) also illustrate reinitiation and the resulting impact on the Rcurve (Chan et al., 2000). In addition, Qiao and Argon (2003a,b) note the importance of bridging along the crack front in iron-silicon alloys.

The focus of the current work is to determine the grain properties required to maintain an intergranular path in a brittle solid. We provide the reader with a background of the grain bridging process and the cohesive zone framework for prediction. Prior to examining multiple modes of propagation, we illustrate that shielding is a natural outcome of a cohesive zone approach to fracture. The competition between transgranular and intergranular fracture for particular crack configurations reveals the role of grain strength, grain toughness, and grain angle. Curves of critical driving force and the resulting surfaces of intergranular fracture in normalized grain strength, normalized grain toughness, and grain angle delineate "strong" and "weak" grains and illustrate the abrupt transition to intergranular fracture. For infinitesimal deformations, we find that linear elastic fracture mechanics is a special case of a cohesive approach to fracture. In addition to documenting thought experiments of candidate microstructures that span the noted transitions, an application section summarizes current findings and places the theoretical studies in the context of structural ceramics.

\section{Background}

In previous work, we proposed that the grain bridging process, illustrated in Figure 1, can be partitioned into five regimes: propagate $J \rightarrow J^{p}$, kink $J^{p} \rightarrow$ $J^{k}$, arrest $J^{k} \rightarrow J^{a}$, stall $J^{a} \rightarrow J^{s}$, and bridge $J^{s} \rightarrow J^{b}$ (Foulk III et al., 2007). These findings were based on an idealized model of an inclined grain bridging

* Although observed experimentally in many ceramics, e.g., Yuan et al. (2003), the notion of crack initiation ahead of a main crack tip is at first sight unexpected in a brittle material where, with the absence of plasticity, the local stresses peak essentially at the crack tip. 
two adjacent grains and the presumption of intergranular fracture. We note that the driving force required for bridge formation $J^{b}$ is much greater than the driving force required to kink $J^{k}$. This marked increase in toughness prior to bridge formation is enabled through an arrest condition $J^{a}$ and a subsequent regime termed stall $J^{a} \rightarrow J^{s}$. During stall, the crack grows incrementally under mounting far-field loading. The intergranular path constrains the crack to grow backwards along the flank of the inclined grain. Depending on the grain angle, the crack will continue to climb (backwards) or reinitiate ahead of the primary crack. Reinitiation is the dominant mechanism for bridge formation.

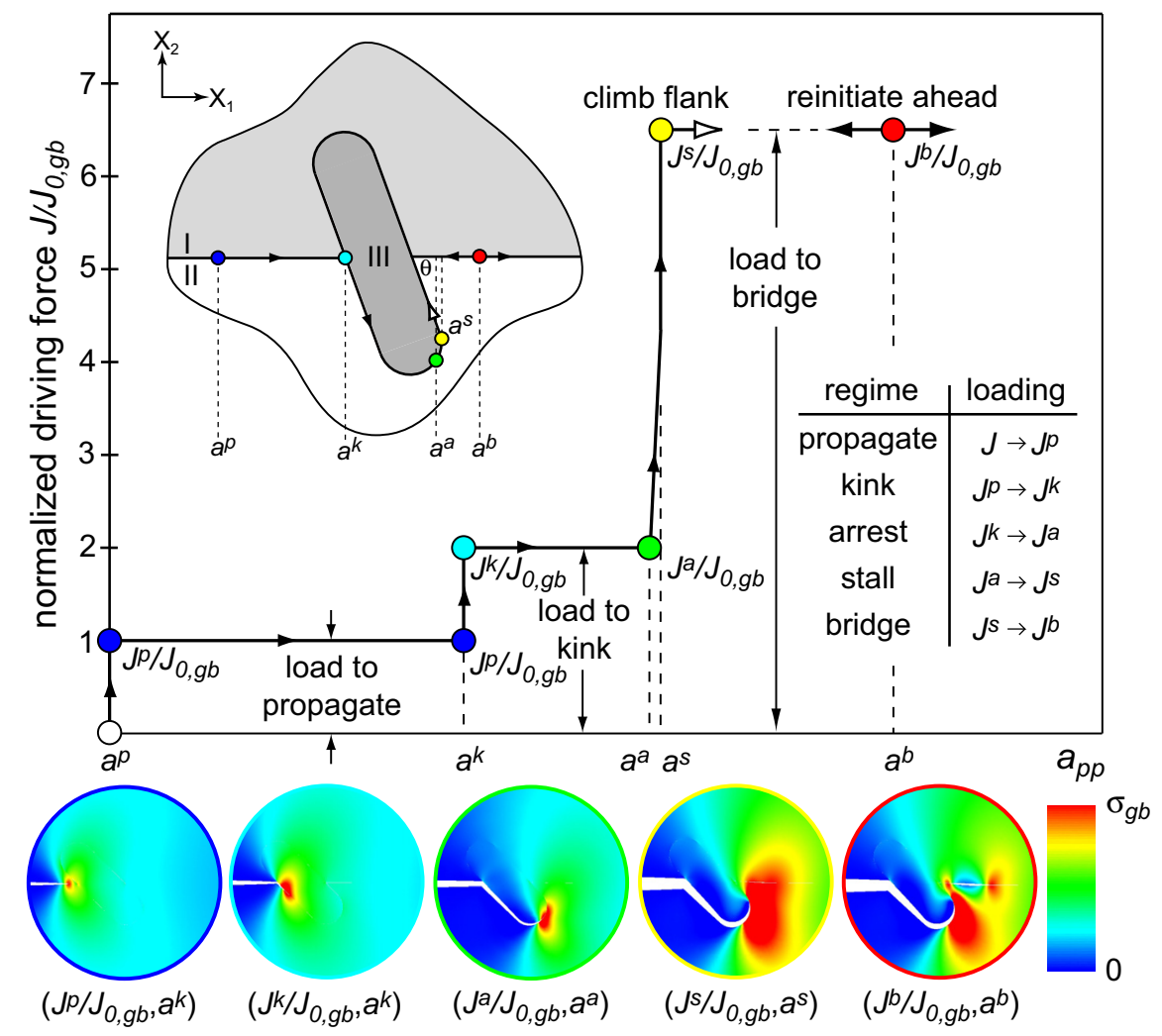

Fig. 1. Partition of the grain bridging process into propagate, kink, arrest, stall, and bridge. The normalized driving force $J / J_{0, g b}$ is plotted against a direct projection of the crack tip $a_{p p}$. Endpoints of the loading regimes are illustrated with $\sigma_{22}$ varying from 0 to the grain boundary strength, $\sigma_{g b}$ (Foulk III et al., 2007).

Although the path of propagation is in general accord with experimental observations, prior studies noted elevated grain stresses peak in the kink and stall regimes. For a grain boundary strength of $\sigma_{g b}=E / 30$, local regions adjacent to the grain boundary are subjected to principal stresses between $E / 12$ and $E / 6$, where $E$ is Young's modulus. Although the noted principal stresses can be justified through observation, we are burdened with quantifying the grain properties needed to maintain an intergranular path. For a given grain boundary strength $\sigma_{g b}$ and toughness $J_{0, g b}$, we seek to determine the grain strength $\sigma_{g}$ and toughness $J_{0, g}$ needed to maintain intergranular fracture. Moreover, in 
accordance with prior findings, we investigate changes in the inclination of the grain, angle $\theta$, to the crack.

We align transgranular/intergranular fracture in the kink regime with the wellstudied problem of a crack penetrating or deflecting when impinging on an interface. We refer the reader to Parmigiani and Thouless (2006) for a review of strength, toughness, and cohesive approaches to penetration/deflection at $\theta=90^{\circ}$. Early studies by Cotterell and Rice (1980), and Faber and Evans (1983) examined the increased toughness due to the deflection of the crack. Further work to quantify the role of modulus mismatch at interfaces was conducted by He and Hutchinson (1989) and Hutchinson and Suo (1991). As noted in Hutchinson and Suo (1991), the driving force is calculated for a putative crack segment ahead or inclined $\theta$ to the pre-crack. Provided the crack segment is small compared to the dimensions of the body, the local stress intensities and resulting driving forces for the grain $j_{g}$ or grain boundary $j_{g b}$ are considered valid. Under increased far-field loading, the direction of propagation is dictated by which condition, $j_{g}=J_{0, g}$ or $j_{g b}=J_{0, g b}$, is satisfied first. An analog would be to insert cohesive zone elements directly ahead or inclined to the pre-crack. Provided the cohesive zone size is small compared to the dimensions of the body (Rice, 1968), the crack will propagate when $j_{g}=J_{0, g}$ or $j_{g b}=J_{0, g b}$, irrespective of $\sigma_{g}$ or $\sigma_{g b}$ for infinitesimal deformations. In finite deformation, increases in $\sigma_{g b}$ promote local grain boundary rotations and reduce the macroscopic driving force required for deflection.

If we enable both modes of propagation via transgranular and intergranular cohesive surfaces, the ratios of grain to grain boundary strength and toughness influence the mode of propagation. Using a cohesive approach, the aforementioned work of Parmigiani and Thouless (2006) generated curves of deflection for varying fracture length scales and modulus mismatch in $\left(\sigma_{g} / \sigma_{g b}, J_{0, g} / J_{0, g b}\right)$ and noted an asymptote in $\sigma_{g} / \sigma_{g b}$ below which penetration was guaranteed. Rather than span many material systems for a finite geometry, we investigate an infinite crack in a high-strength, low toughness material. Within the context of structural ceramics, we do not observe asymptotic behavior in $\sigma_{g} / \sigma_{g b}$. Moreover, decreases in grain angle result in deflection at reduced $\sigma_{g} / \sigma_{g b}$.

Studies examining the competition between intergranular and transgranular fracture not only yield the grain properties needed to maintain the prior partition of grain bridging but also provide insight into the mechanics of transition. A fundamental understanding of the transition to transgranular fracture is crucial. Maintaining an intergranular fracture path (which includes crack reinitiation) in brittle materials is essential for generating a rapid rise in the resistance curve which governs the macroscopic strength at realistic flaw sizes. 


\section{Cohesive zone framework for fracture}

To investigate transitions to transgranular fracture, we employ the finite element method and prescribe constitutive models governing bulk deformation and surface separation (Needleman, 1987, 1990; Tvergaard and Hutchinson, 1990, 1992). We refer the reader to Klein et al. (2001) for a review of early works. Because the length scales associated with modeling transgranular/intergranular fracture in brittle microstructures are on the order of $\mathrm{nm}$ and specimen geometries are on the order of $\mathrm{cm}$, we examine 2-D geometries (in plane strain) and employ a $K$-field boundary condition. In addition, we note that if the nonlinear region at the macroscopic crack tip is contained, we have an accurate representation of the far-field driving force; indeed, we prescribe it.

A schematic of the model for investigating transgranular/intergranular fracture is illustrated in Figure 2. A mode I, $K$-field displacement boundary condition is applied to a $50 \mu \mathrm{m}$ disk containing three grains (I, II, III). To remain consistent with prior work, the grains (I, II, III) are assumed to be elastic, isotropic, and spatially uniform. These simplifying assumptions permit fundamental numerical studies that increase our understanding of local crack-tip processes. We note that in contrast to metals, the measured toughness of ceramic systems that exhibit transgranular fracture is relatively insensitive to crystallographic orientation (Ballarini et al., 2001). The assumptions of isotropy and direct penetration $\left(K_{I I}=0\right)$, while simplified, are reasonable for initial studies.

We also assume that the grain boundaries are spatially uniform and do not vary between grains (I-III, II-III). The idealized microstructure is pre-cracked to the inclined grain boundary and the macroscopic crack length $a$ (employed by the boundary condition) is assumed to be constant. We place cohesive

surface elements along the grain boundaries and within the grain to simulate intergranular and transgranular fracture, respectively. We acknowledge, as in prior findings, that although reinitiation is a three-dimensional process subject to variations in geometry and material properties, the current two-dimensional framework is especially applicable to the interlocking, plate-like grains of $\mathrm{SiC}$ (Gilbert et al., 1996).

In an attempt to clarify the role of the cohesive zone, we make a distinction between cohesive zone initiation and growth, and crack initiation and growth. The cohesive zone initiates when cohesive surface elements achieve peak strength. Under increased far-field loading, the cohesive zone grows. Cohesive zone growth is termed formation and is a phenomenological representation of the failure process. Growth continues until the cohesive zone reaches a length $l_{c z}$ in which the tail of the cohesive zone is unloaded and the far-field, 


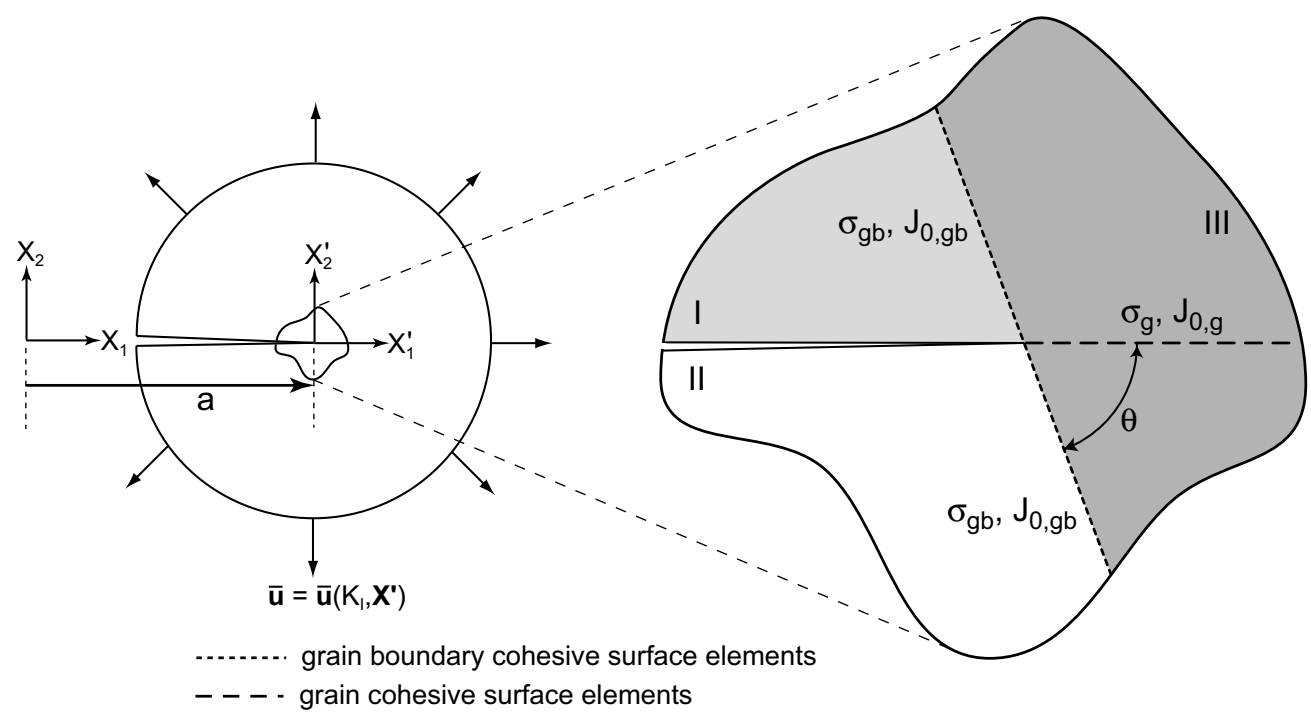

Fig. 2. An idealized two-dimensional framework for investigating transitions to transgranular fracture. $K$-field boundary conditions are applied to a disk containing three grains (I, II, III). Cohesive surface elements seeded within the inclined grain (III) and along the grain boundaries enable transgranular and intergranular fracture.

mode I driving force is equivalent to the input toughness (for $\theta=0$ ). We associate a cohesive zone of length $l_{c z}$, the material process zone size, with crack initiation and the translation of the cohesive zone with crack growth. In this work, crack initiation and growth are combined into crack propagation.

Although the paths of propagation are selected a priori, our goal is to adequately resolve and thoroughly understand the competition between intergranular and transgranular fracture for particular crack configurations in the evolution of grain bridging. For a constant grain boundary strength $\sigma_{g b}$ and toughness $J_{0, g b}$, we vary the grain strength $\sigma_{g}$, toughness $J_{0, g}$, and angle $\theta$.

\subsection{Grain and grain boundary constitutive models}

We employ relatively simple grain (bulk) and grain boundary (surface) models having few material parameters. Bulk deformation is governed by a hyperelastic model proposed by Simo et al. (1985), Simo and Hughes (1997). The resulting Cauchy stress tensor $\boldsymbol{\sigma}$ is

$$
\boldsymbol{\sigma}=\frac{1}{\operatorname{det} \mathbf{F}}\left[\left(\frac{\kappa}{2}\left[(\operatorname{det} \mathbf{F})^{2}-1\right]-\frac{\mu}{3} \operatorname{tr} \overline{\mathbf{b}}\right) \mathbf{I}+\mu \overline{\mathbf{b}}\right] .
$$

where $\kappa$ is the bulk modulus, $\mu$ is the shear modulus, $\mathbf{F}$ is the deformation gradient, and $\overline{\mathbf{b}}=(\operatorname{det} \mathbf{F})^{-2 / 3} \mathbf{b}$ is the isochoric part of the left Cauchy-Green stretch tensor $\mathbf{b}(\operatorname{det} \overline{\mathbf{b}}=1)$. We note that for small, deformations, the bulk 
model reduces to isotropic, linear elasticity and permits a consistent application of the $K$-field displacement boundary condition.

The constitutive model governing grain and grain boundary fracture is taken from Tvergaard and Hutchinson (1993). The normal $t_{n}$ and tangential $t_{t}$ components of the traction vector are

$$
t_{n}(\boldsymbol{\Delta})=\frac{\sigma(\lambda)}{\lambda}\left(\frac{\Delta_{n}}{\delta_{n}}\right), \quad t_{t}(\boldsymbol{\Delta})=\frac{\sigma(\lambda)}{\lambda} \frac{\delta_{n}}{\delta_{t}}\left(\frac{\Delta_{t}}{\delta_{t}}\right)
$$

where $\boldsymbol{\Delta}$ is the gap vector, $\lambda$ is the generalized displacement

$$
\lambda=\sqrt{\left(\Delta_{n} / \delta_{n}\right)^{2}+\left(\Delta_{t} / \delta_{t}\right)^{2}}
$$

$\sigma(\lambda)$ is the traction-generalized displacement law containing a peak strength $\sigma_{\max }$, and $\delta_{n}$ and $\delta_{t}$ are the characteristic length scales governing normal and tangential separation. The normal and tangential openings are defined as $\Delta_{n}=$ $\boldsymbol{\Delta} \cdot \mathbf{n}$ and $\Delta_{t}=\boldsymbol{\Delta} \cdot \mathbf{t}$ with respect to the local normal $\mathbf{n}$ and tangent $\mathbf{t}$ to the cohesive surface element.

The normalized traction-generalized displacement model for grain and grain boundary separation is assumed to be triangular with $\sigma_{\max }$ at $\lambda=0.02$. We postulate that the normal strength is an upper bound for the shear strength and equate the characteristic length scales to find $\tau_{\max }=\sigma_{\max }$. The work of separation (toughness) is independent of mode-mixity. Simplifications in both the model and chosen parameters result in two material constants, strength and toughness. Simulations require specification of four parameters: grain strength $\sigma_{g}$, grain toughness $J_{0, g}$, grain boundary strength $\sigma_{g b}$, and grain boundary toughness $J_{0, g b}$. For this work, we postulate that the traction-generalized displacement model is reversible. We do not stipulate an additional model for unloading because we cannot exclude grain or grain boundary healing.

\subsection{Material properties and discretization}

For the quasi-static simulations presented here, the chosen material properties are reflective of a high strength, low toughness material system. Although the finite element framework does admit distributions of material properties for both the grains and grain boundaries, initial studies on homogeneous systems provide the requisite baseline for future work. Properties for the model system, akin to silicon nitride, mirror our prior study on intergranular fracture (Foulk III et al., 2007). The Young's modulus E and Poisson's ratio $\nu$ are $303 G P a$ and 0.21 , respectively. For all simulations, the grain boundary strength $\sigma_{g b}$ is $E / 30$ and the grain boundary toughness $J_{0, g b}$ is $40 \mathrm{~J} / \mathrm{m}^{2}$. The 
grain strength $\sigma_{g}$ ranges from $E / 15$ to $E / 7\left(2.0 \sigma_{g b} \rightarrow 4.3 \sigma_{g b}\right)$ and the grain toughness varies from $40 \mathrm{~J} / \mathrm{m}^{2}$ to $400 \mathrm{~J} / \mathrm{m}^{2}\left(J_{0, g b} \rightarrow 10 J_{0, g b}\right)$.

A typical mesh used for this study is illustrated in Figure 3. The radius of the disk is $50 \mu \mathrm{m}$ and the cohesive surface element size $h$ is $0.5 \mathrm{~nm}$. Prior studies employed a $2.5 \mathrm{~nm}$ cohesive surface element size for intergranular fracture. The element size is decremented in this study $(h \leq 0.5 \mathrm{~nm})$ to adequately resolve the transgranular cohesive zone and promote numerical stability. Mesh refinement studies are conducted to ensure convergent solutions. We note that no matter the element size, we always employ a local continuum description. The element size was not selected to resolve features on the order of $h$ and the only relevant length scale in the simulation, the cohesive zone size, is typically greater than $8 \mathrm{~nm}$ and permits a continuum description. To be consistent with prior work, the cohesive zone size is defined as the distance from the peak $\max \left(t_{n}\right)$ to the tail $\sim 0.1 \max \left(t_{n}\right)$ of the global traction distribution.

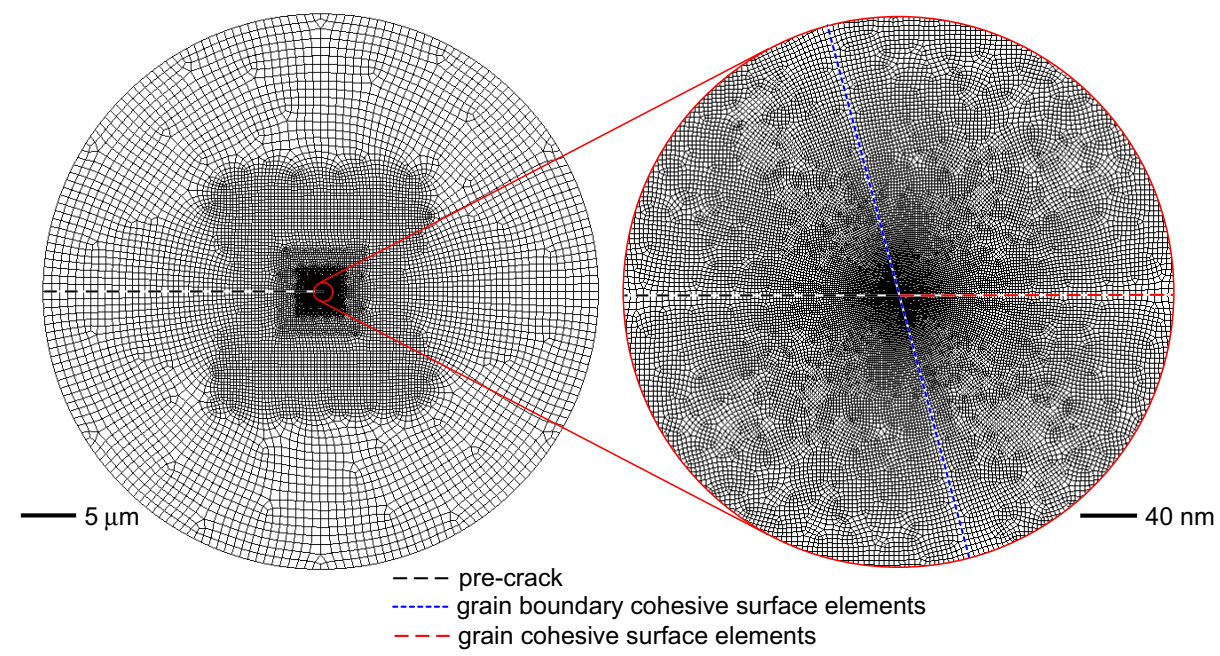

Fig. 3. Typical mesh employed in the analysis. The radius of the disk is $50 \mu \mathrm{m}$ and the cohesive surface element size $h$ at the crack tip is $0.5 \mathrm{~nm}$.

\section{Crack shielding in a cohesive framework}

Because multiple crack tips compete through shielding, it is important to develop an understanding of crack shielding in the context of cohesive zone modeling. To achieve this, we idealize Figure 2 and consider a single path of propagation, transgranular or intergranular fracture. Specifically, local crack tip fields are compared for a sharp crack and a cohesive zone. As real solids cannot support singular fields, we compare with singular fields to determine how the stresses are redistributed when the singularity is relieved through the introduction of a cohesive zone. Rather than focus on stresses adjacent to the 
cohesive zone for a particular mode of propagation, we investigate the impact on the other mode of propagation. A qualitative understanding of shielding is sought through an examination of the stresses available to overcome the cohesive strength in the competing mode of propagation.

For a particular grain boundary strength $\sigma_{g b}=10 \mathrm{GPa}$ and toughness $J_{0, g b}=$ $40 \mathrm{~J} / \mathrm{m}^{2}$, the grain strength $\sigma_{g}$ and toughness $J_{0, g}$ governing transgranular fracture are $2.1 \sigma_{g b}$ and $3.61 J_{0, g b}$, respectively. The transgranular toughness was selected to coincide with the kink (deflection) toughness $J^{k}$ for $\theta=90^{\circ}$. Consequently, both penetration or deflection will occur at the same far-field driving force $J_{c}=J_{0, g}=J^{k}\left(J_{0, g b}, 90^{\circ}\right)$. Moreover, at propagation, the fields governing both modes rapidly decay to the same far-field values. Although the far-field quantities are identical for both cases, Figure 4 and Figure 6 tell very different stories.

\subsection{Grain boundary shielding}

Figure 4 illustrates transgranular fracture within a cohesive framework and highlights the grain boundary. If we monitor $\sigma_{11}$ along the grain boundary, the maximum occurs at a normalized loading of $J / J_{0, g}=0.27$. At $J / J_{0, g}=0.27$, almost the entire cohesive zone is at the grain strength $\sigma_{g}$ and differences between the sharp crack and cohesive zone fields are minimal. Subsequent unloading and growth occurs until the cohesive zone reaches $l_{c z}$ at propagation $J=J_{0, g}$.

At propagation $J=J_{0, g}$, the fields are distinctly different. The redistribution of $\sigma_{22}$ through the introduction of a finite strength impacts both $\sigma_{12}$ and $\sigma_{11}$. Peak values of $\sigma_{12}$ and $\sigma_{11}$ reduce and shift with the peak traction $\sigma_{g}$ in the cohesive zone. Stresses necessary for grain boundary initiation $\left(\sigma_{11}, \sigma_{12}\right)$ are a fraction of the maximum $J / J_{0, g}=0.27$. Differences in $\sigma_{11}$ along the grain boundary for $J / J_{0, g}=0.27$ and $J / J_{0, g}=1$ are illustrated in Figure 5a. Arc length $s$ along the grain boundary is normalized by the transgranular cohesive zone length $l_{c z}=66 \mathrm{~nm}$. For $J / J_{0, g}=0.27$, the stress redistribution is minimal and confined to fractions of $s / l_{c z}$. In contrast, the curves from the sharp crack and cohesive zone solutions diverge for $J / J_{0, g}=1$. Although we make no attempt to quantify the local driving force for intergranular fracture, we can readily associate shielding with $\sigma_{11}$ along the grain boundary. Cohesive zone formation (unloading and growth) in the transgranular mode of propagation shields the grain boundary from the stresses required for cohesive zone initiation in the intergranular mode of propagation.

To investigate the effect of grain strength, we sample $\sigma_{11}$ at $s / l_{c z}=0$ for varying $\sigma_{g} / \sigma_{g b}$ holding $J_{0, g}$ constant. The resulting curves are plotted in Figure 5b. 


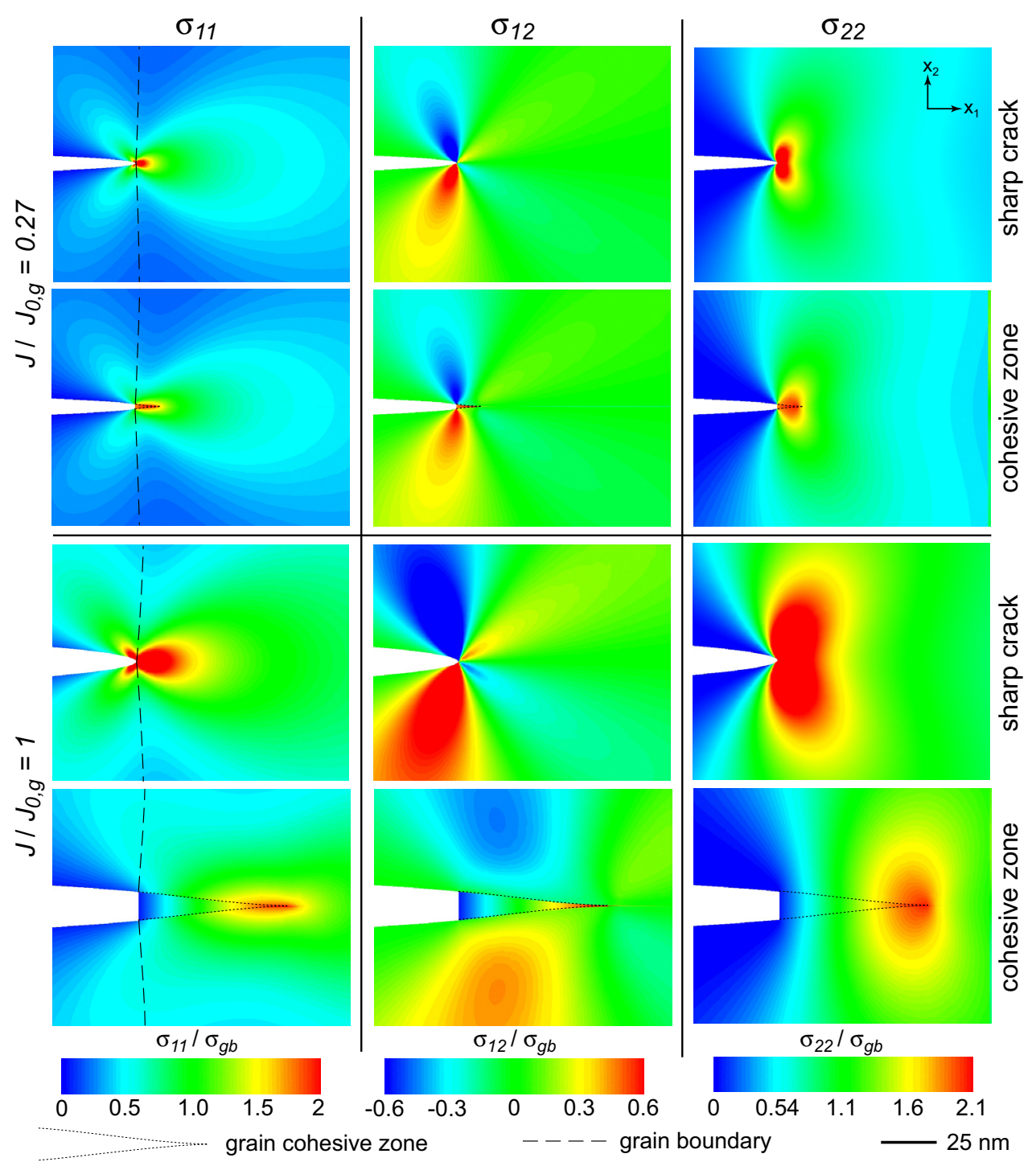

Fig. 4. The redistribution of $\sigma_{11}, \sigma_{12}$, and $\sigma_{22}$ caused by the introduction of a transgranular cohesive zone with strength $2.1 \sigma_{g b}$ and toughness $J_{0, g}=J^{k}\left(J_{0, g b}, 90^{\circ}\right)$. Cohesive zone formation within the grain shields the grain boundary from initiation stresses $\left(\sigma_{11}, \sigma_{12}\right)$. The first configuration $J / J_{0, g}=0.27$ is taken at peak $\sigma_{11}$ along the grain boundary and the second configuration $J / J_{0, g}=1.0$ is taken at transgranular propagation. Contours of the normal traction in the cohesive zone mirror $\sigma_{22}$.

As expected, increasing $\sigma_{g}$ raises the peak $\sigma_{11}$. In addition, we note that an increase in $\sigma_{g}$ at constant $J_{0, g}$ decreases $l_{c z}$. For normalized cohesive strengths $\sigma_{g} / \sigma_{g b}$ of $3.3,3.0,2.7,2.3,2.1$, and 2.0 , the cohesive zone size $l_{c z}$ is $29,34,41$, $52,60,66$, and $78 \mathrm{~nm}$, respectively. Decreasing $l_{c z}$ lessens the shift of the peak traction $\sigma_{g}$ in the transgranular cohesive zone and promotes a broadening of $\sigma_{11}$ required for intergranular cohesive zone initiation. 

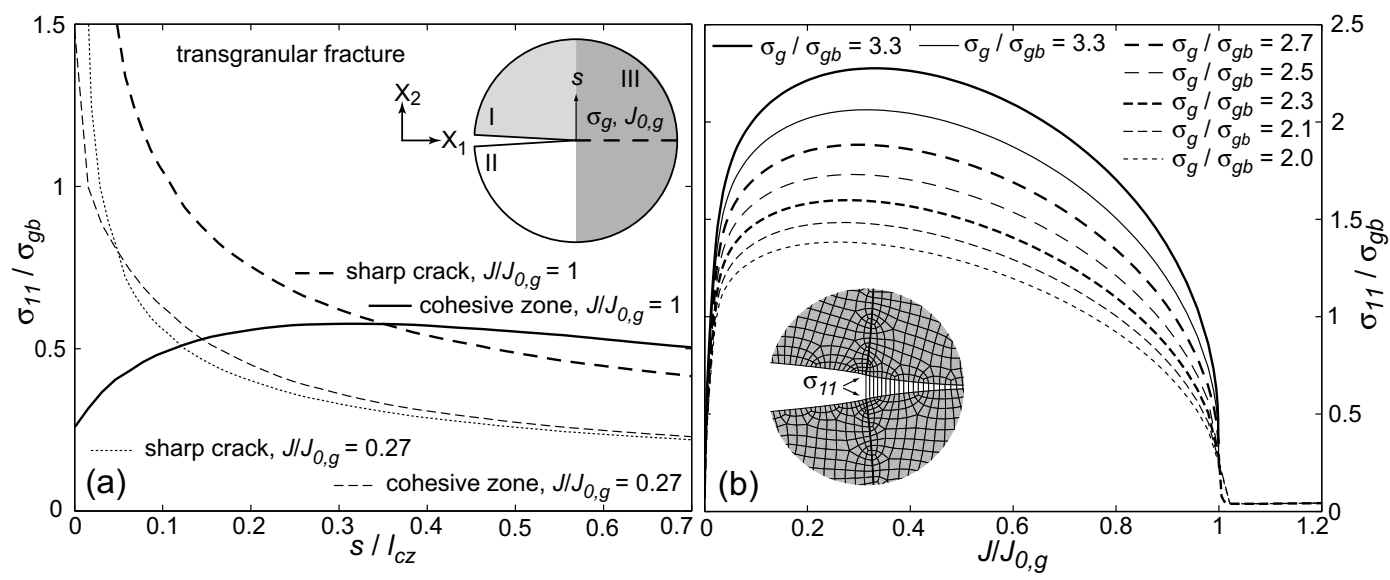

Fig. 5. Variance of $\sigma_{11} / \sigma_{g b}$ with (a) normalized length $s / l_{c z}$ along the grain boundary for $\sigma_{g}=2.1 \sigma_{g b}$ and $J_{0, g}=J^{k}\left(J_{0, g b}, 90^{\circ}\right)$ and (b) far-field loading $J / J_{0, g}$ at $s / l_{c z}=0$ for varying normalized grain strength $\sigma_{g} / \sigma_{g b}$. Solutions for the sharp crack and cohesive zone diverge at transgranular propagation $J / J_{0, g}=1$. For constant toughness, increases in strength elevate and broaden peaks for intergranular cohesive zone initiation.

\subsection{Grain shielding}

In contrast, Figure 6 illustrates intergranular fracture within a cohesive framework and highlights the grain. If we monitor $\sigma_{22}$ within the grain, the maximum occurs at a normalized loading of $J / J_{0, g}=0.31$. At $J / J_{0, g}=0.31$, almost the entire cohesive zone borders the grain strength under mixed-mode loading and differences between the sharp crack and cohesive zone fields are minimal. At propagation $J=J^{k}=J_{0, g}$, the fields are again, distinctly different. Unloading on the grain boundary redistributes local stresses and limits $\sigma_{11}$, $\sigma_{12}$, and the gradients the body can support. Without substantial shear gradients, the stress concentration in $\sigma_{22}$ cannot be supported $\left(\sigma_{12,1}+\sigma_{22,2}=0\right)$. Consequently, the stresses in the grain approach uniformity with increasing intergranular cohesive zone growth. The redistribution is illustrated in Figure 7a for $J / J_{0, g}=0.31$ and $J / J_{0, g}=1$. Arc length along the grain is normalized by $l_{c z}=75 \mathrm{~nm}$. When $\sigma_{22}$ reaches the maximum at $J / J_{0, g}=0.31$, minimal differences between the sharp crack and the cohesive zone exist and the redistribution is limited to small values of $s / l_{c z}$. However, like the prior case, the curves diverge at propagation $J / J_{0, g}=1$. Intergranular cohesive zone formation shields the grain from the elevated stresses needed for transgranular cohesive zone initiation.

In addition to investigating grain shielding for the selected grain boundary strength and toughness at a particular configuration, we also seek to obtain an understanding of the effect of grain angle $\theta$. Figure $7 \mathrm{~b}$ illustrates the effect of grain angle on $\sigma_{22} / \sigma_{g b}$ at $s / l_{c z}=0$. For decreasing $\theta$, intergranular cohesive zones readily initiate, grow, and effectively shield the grain. Both the peak and 


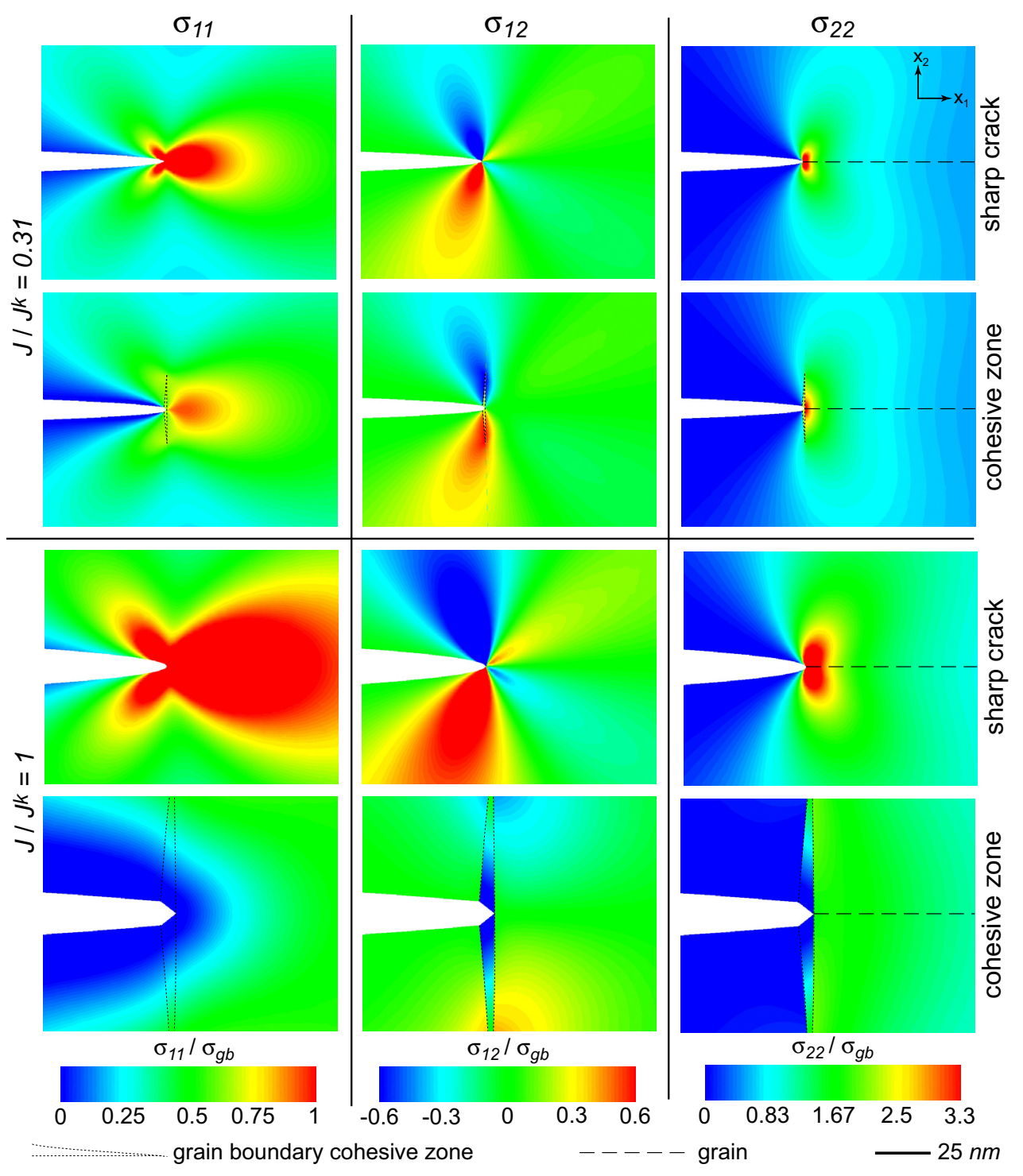

Fig. 6. The redistribution of $\sigma_{11}, \sigma_{12}$, and $\sigma_{22}$ caused by the introduction of an intergranular cohesive zone with strength $\sigma_{g b}$ and toughness $J_{0, g b}$. Cohesive zone formation on the grain boundaries shields the grain from initiation stresses $\left(\sigma_{22}\right)$. The first configuration $J / J_{0, g}=0.31$ is taken at peak $\sigma_{22}$ within the grain and the second configuration $J / J_{0, g}=1.0$ is taken at intergranular propagation. Contours of the normal traction in the cohesive zone mirror $\sigma_{11}$.

broadness of $\sigma_{22}$ reduce with decreasing $\theta$. Because the driving force required to kink is a function of grain angle, each curve is terminated at $J^{k}$.

Although the fields between a sharp crack and a cohesive zone approach for transgranular or intergranular fracture are similar for $J / J_{c} \sim 0.3\left(K / K_{c} \sim\right.$ $0.5)$, marked differences exist at $J / J_{c}=1$. The introduction of a finite strength and toughness not only relieves the singularity but also shields the competing mode (transgranular/intergranular) of propagation. Shielding is quantified as a reduction in the stresses available for cohesive zone initiation in the competing 


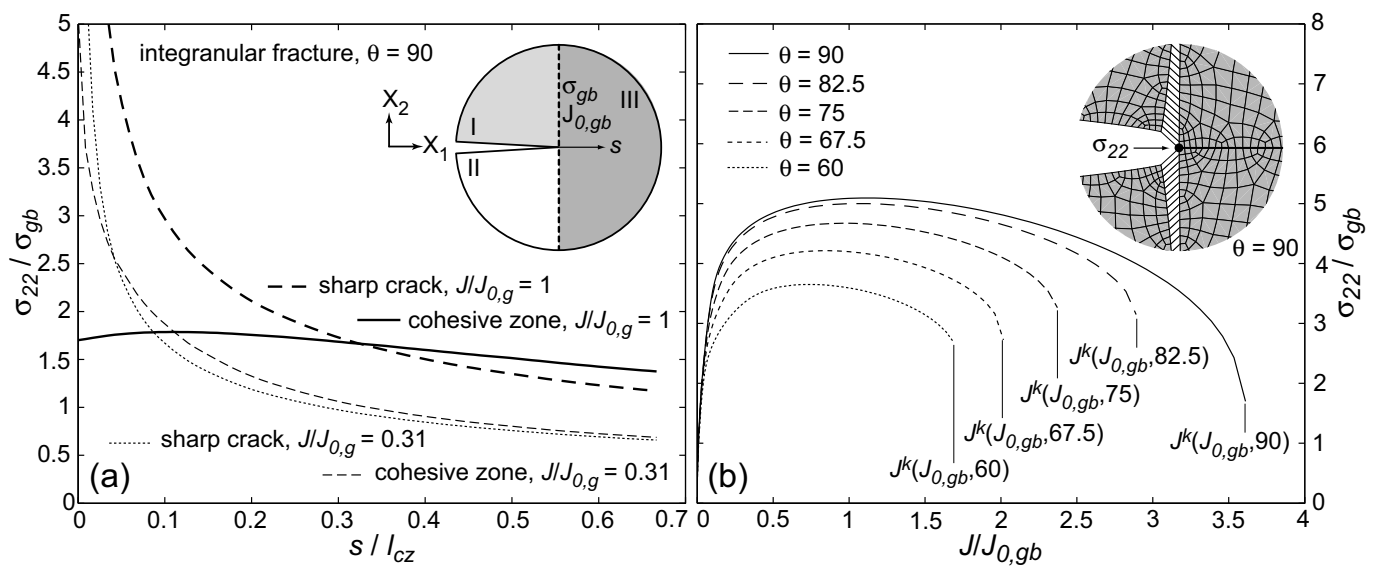

Fig. 7. Variance of $\sigma_{22} / \sigma_{g b}$ with (a) normalized length $s / l_{c z}$ within the grain and (b) far-field loading $J / J_{0, g b}$ at $s / l_{c z}=0$ under decreasing grain angle $\theta$ for a grain boundary with prescribed strength $\sigma_{g b}$ and toughness $J_{0, g b}$. Solutions for the sharp crack and cohesive zone diverge at intergranular propagation $J / J_{0, g}=1$. At lower grain angles, intergranular cohesive zones readily initiate and grow to effectively shield the grain.

mode. For transgranular fracture of a grain at $90^{\circ}$, decreasing $\sigma_{g}$ at constant $J_{0, g}$ increases the transgranular cohesive zone size $l_{c z}$ and results in a decrease in the stresses available for intergranular cohesive zone initiation for all $J / J_{0, g}$. Intergranular fracture under decreasing $\theta$ confirms that the initiation stresses available for transgranular cohesive zone initiation are mitigated for all $J / J_{0, g b}$. We emphasize that the fields at propagation for transgranular fracture, Figure 4, and intergranular fracture, Figure 6, rapidly decay to identical fields; both cases propagate at the same far-field loading $J_{c}=J_{0, g}=J^{k}\left(J_{0, g b}, 90^{\circ}\right)$. The nonlinear regions within the $K$-annulus, however, are entirely different.

Shielding is a natural outcome of a cohesive zone approach to fracture. The degree of shielding is influenced by the cohesive strength and the cohesive zone size. Although there is not a direct correspondence between the results of single path solutions of transgranular or intergranular fracture and the competition between multiple modes involving stable and unstable propagation, an enhanced understanding of shielding in the context of a cohesive zone approach is integral to interpreting transitions to transgranular fracture.

\section{Competition between transgranular and intergranular fracture}

To obtain a greater understanding of transitions to transgranular fracture, we revisit the penetration/deflection problem illustrated in Figure 2 and enable both modes of propagation. At grain angles $\theta$ of $90^{\circ}, 82.5^{\circ}, 75^{\circ}, 67.5^{\circ}$, and $60^{\circ}$, a series of simulations are conducted at normalized grain strengths $\sigma_{g} / \sigma_{g b}$ of 
2.0, 2.1, 2.3, 2.5, 2.7, 3.0, and 3.3. At each grain strength, the grain toughness is varied from $J_{0, g b}$ to $10 J_{0, g b}$ in increments of $J_{0, g b}$. The critical driving force $J_{c}$ and mode of propagation (intergranular, transgranular) are noted. Three subsequent bisections of the critical driving forces bordering the mode change are employed to find the transgranular $\rightarrow$ intergranular transition within $J_{0, g b} / 8$. Through a variation in grain geometry and material properties, we seek to determine the grain angle, strength, and toughness required to maintain intergranular fracture.

The family of simulations in $\left(\sigma_{g} / \sigma_{g b}, J_{0, g} / J_{0, g b}, \theta\right)$ for constant $\sigma_{g b}$ and $J_{0, g b}$ not only gives the requisite grain properties to maintain an intergranular path but also sheds light on the mechanics of transition. To illustrate the fundamental differences in the evolution of transgranular and intergranular fracture, we include Figure 8 and Figure 9. Both simulations employ a common transgranular toughness, $J_{0, g}=J_{0, g b}$. The first case, Figure 8, employs a grain strength $\sigma_{g}$ of $2.5 \sigma_{g b}$ while the second case, Figure 9, employs a grain strength $\sigma_{g}$ of $3.3 \sigma_{g b}$.
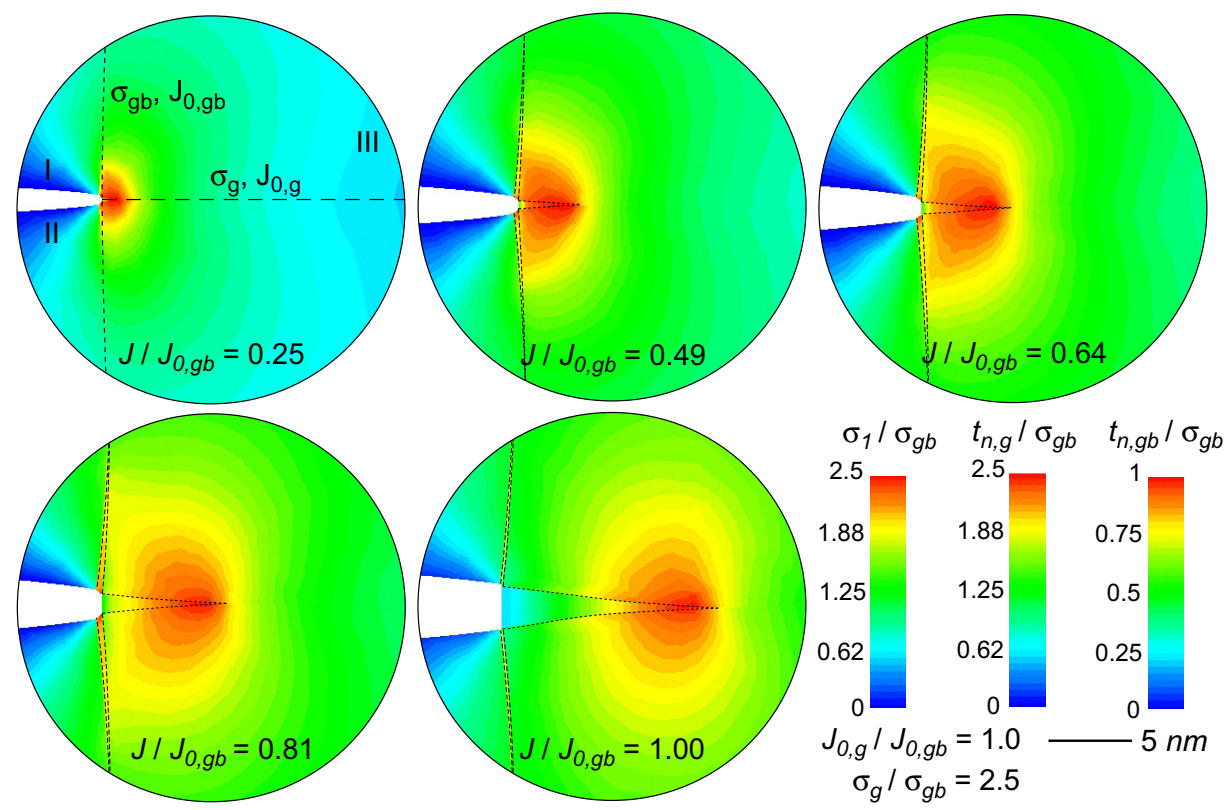

Fig. 8. Transition to transgranular fracture for $\sigma_{g} / \sigma_{g b}=2.5$ and $J_{0, g} / J_{0, g b}=1$. The first principal stress $\sigma_{1}$ and the normal traction within the grain $t_{n, g}$ and on the grain boundary $t_{n, g b}$ illustrate the competing cohesive zones. Dotted lines are drawn to delineate the cohesive surface elements. The cohesive zone size $l_{c z}$ within the grain is approximately $12 \mathrm{~nm}$.

For the process shown in Figure 8, the grain strength is insufficient to enable significant cohesive zone formation on the grain boundaries. Consequently, the cohesive zone initiates in the grain and the peak traction $\sigma_{g}$ moves into the grain. Fracture occurs at the grain toughness, $J_{0, g}$. 

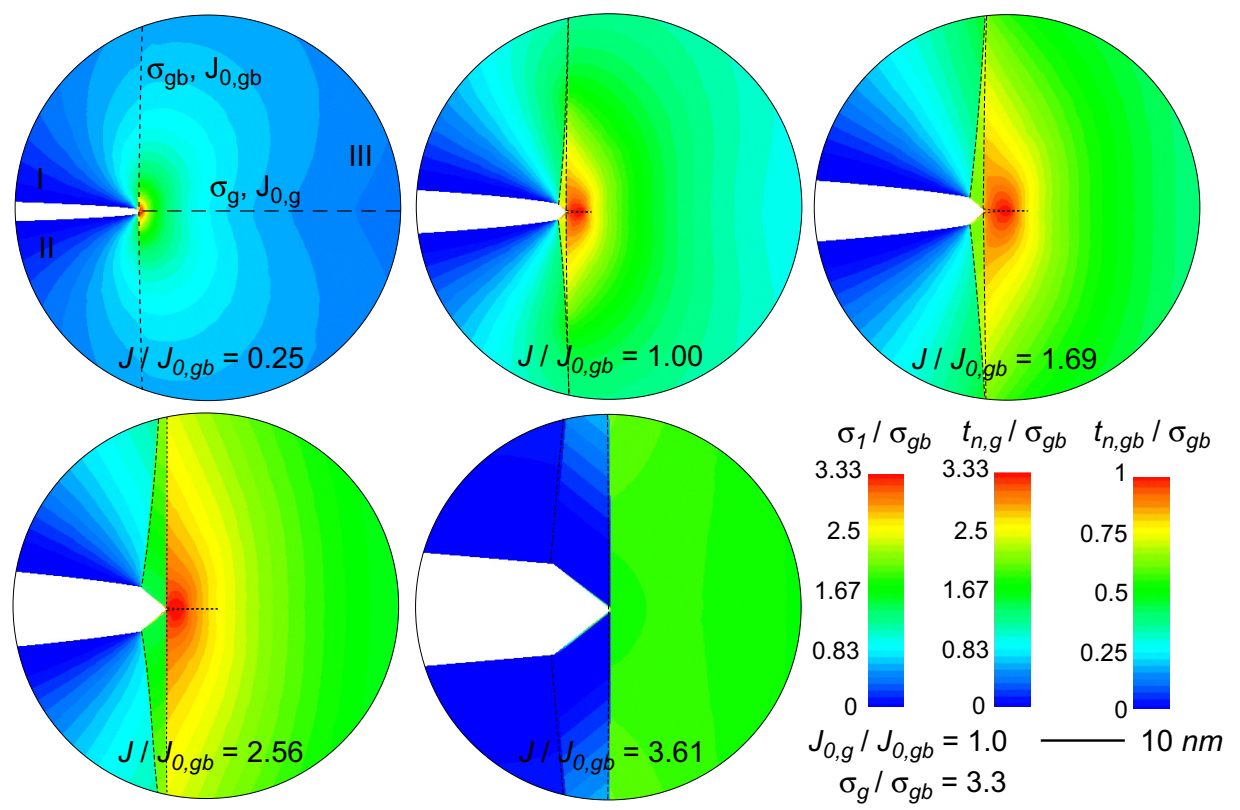

Fig. 9. Continued intergranular fracture for $\sigma_{g} / \sigma_{g b}=3.3$ and $J_{0, g} / J_{0, g b}=1$. The first principal stress $\sigma_{1}$ and the normal traction within the grain $t_{n, g}$ and on the grain boundary $t_{n, g b}$ illustrate the competing cohesive zones. Dotted lines are drawn to delineate the cohesive surface elements. The cohesive zone size $l_{c z}$ on the grain boundary is approximately $75 \mathrm{~nm}$.

In contrast to Figure 8, the fracture process in Figure 9 is altogether different. The stronger grain enables significant cohesive zone formation along the grain boundaries, which in turn, shields the grain. This is evident as the location of the peak stress in the grain cohesive zone actually moves backwards between $J=1.69 J_{0, g b}$ and $J=2.56 J_{0, g b}$. The grain cohesive zone unloads despite increases in the far-field loading. We note that this progression occurs at driving forces much greater than the grain toughness $J_{0, g}$. When the driving force is sufficient to deflect a crack at $90^{\circ}, J=3.61 J_{0, g b}=J^{k}$, the crack kinks.

\subsection{Curves of critical driving force and intergranular fracture}

Employing a single path, linear elastic fracture mechanics (LEFM) methodology, we stipulate that the crack will propagate when $J$ is either the grain boundary toughness $J_{0, g}$ or the kink toughness $J^{k}=J^{k}\left(J_{0, g b}, \theta\right)$. Through the cohesive intergranular solution, one can deduce the required grain toughness for deflection $J_{0, g}=J^{k}$. The transition to intergranular fracture for a single path solution is illustrated in Figure 10a. The diagonal line, $J_{c}=J_{0, g}$, reflects transgranular fracture. The horizontal line, $J_{c}=J^{k}$, indicates intergranular fracture. We note that obtaining the kink toughness prior to $J_{0, g}=J^{k}$ is prohibited. 
If we consider a cohesive multiple path solution, stable propagation will still occur at $J_{0, g}$ or $J^{k}$. However, the mode of propagation is dictated by grain and grain boundary strength and toughness at each grain angle. A schematic of the multiple path construction is illustrated in Figure 10c for three normalized grain strengths $\sigma_{g} / \sigma_{g b}$. At a particular normalized grain strength and normalized grain toughness $J_{0, g} / J_{0, g b}$, stable crack propagation will lie on the diagonal (transgranular) or horizontal (intergranular) line. The intersection of the horizontal and diagonal lines coincides with single path cohesive solutions of transgranular or intergranular fracture for a given grain boundary strength and toughness.

For infinitesimal deformations, Figure 10 will reduce to LEFM and the cohesive transition in Figure $10 \mathrm{~b}$ can be aligned with the energetic transition in Figure 10a. For a particular normalized grain strength, the multiple path cohesive methodology reduces to the single path LEFM methodology. All other normalized strengths yield solutions outside the scope of linear elastic fracture mechanics.

Unstable propagation occurs for a narrow range of material properties that promote intense interaction between the modes of propagation. During the competition between transgranular and intergranular fracture, both cohesive zones are forming (and shielding) under increased far-field loading. Although the mode of propagation will eventually coincide with $\min \left(J^{k}, J_{0, g}\right)$, the driving force $J$ is greater than the resistance $\min \left(J^{k}, J_{0, g}\right)$. Consequently, the quasi-static system is overdriven and globally unstable. Candidate simulations are restarted with implicit dynamics to examine a branch of the solution. Depending on the normalized grain strength $\sigma_{g} / \sigma_{g b}$, unstable transitions to intergranular fracture result in a rapid increase or decrease in the macroscopic toughness.

For "strong" grains (case A) in Figure 10c, transgranular propagation $J_{c}=J_{0, g}$ occurs until a normalized grain toughness $J_{0, g} / J_{0, g b}$ enables a rapid transition to intergranular fracture $J_{c}=J^{k}$. The grain is sufficiently strong and tough to promote grain boundary initiation and growth. Continued growth (and unloading) on the grain boundary shields the grain and enables $J>J_{0, g}$. The critical driving force for propagation is $J^{k}$ where $J^{k}>J_{0, g}$. For each grain angle, there also exists a grain strength (case B) which yields a transition consistent with a single path (transgranular, intergranular) of propagation, $J_{c}=J^{k}=J_{0, g}$. "Weak" grains (case C) enable propagation in a transgranular mode past the kink toughness, $J>J^{k}$. Unloading in the transgranular cohesive zone shields the grain boundary from the stresses required for cohesive zone initiation. Transgranular propagation $J_{c}=J_{0, g}$ occurs until a substantial normalized grain toughness enables a rapid transition to intergranular fracture. The critical driving force for intergranular propagation remains $J^{k}$ where $J^{k}<J_{0, g}$. We note that simulations in the rapid transition to intergranular 

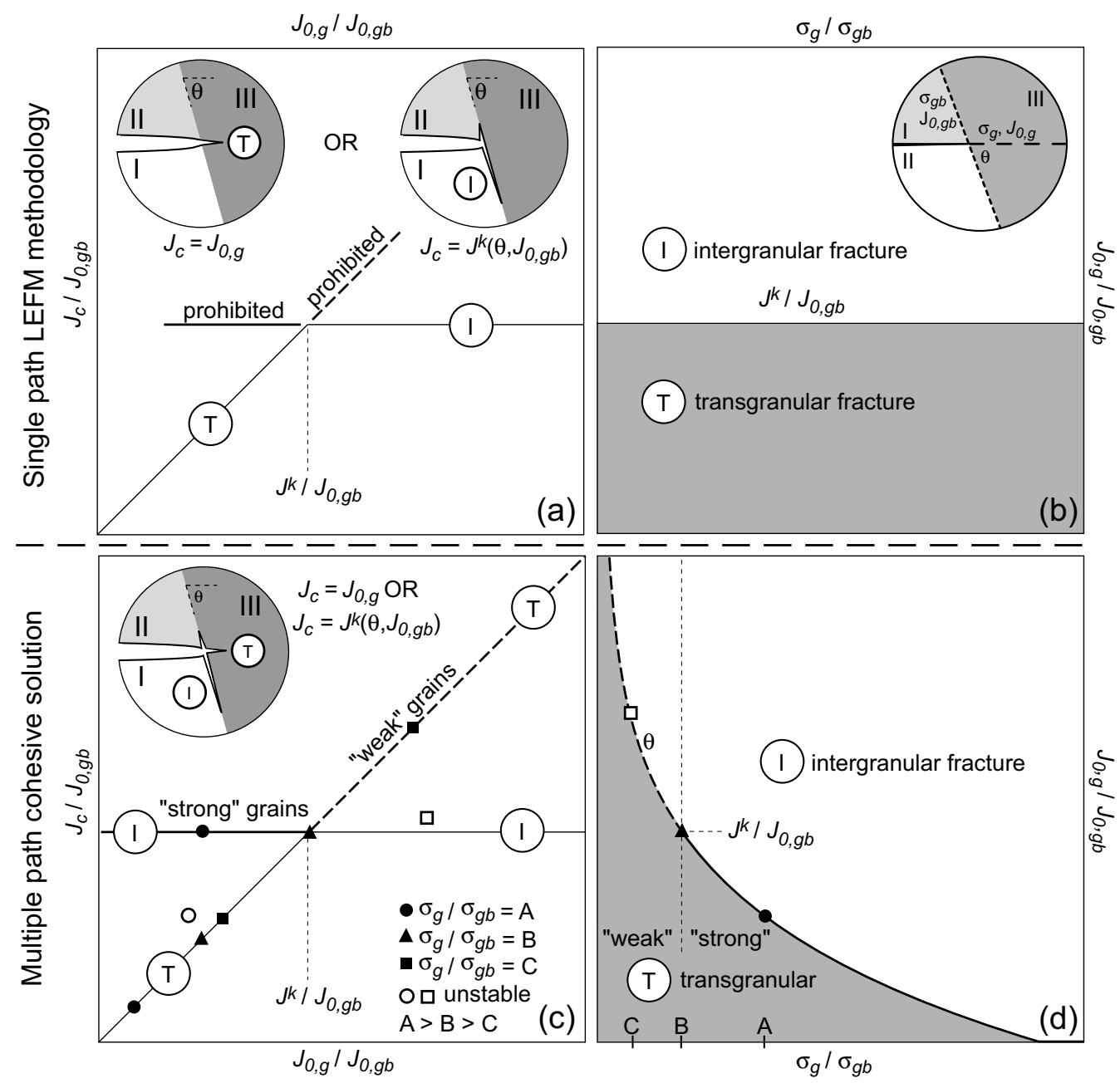

Fig. 10. Schematics of the critical macroscopic driving force $J_{c}$ for (a) single path linear elastic fracture mechanics (LEFM) methodology and (c) multiple path cohesive solutions for intergranular or transgranular fracture. The transitions to intergranular fracture for a series of constant grain strength and varying grain toughness result in a curve of intergranular fracture in (b) $J_{0, g} / J_{0, g b}$ or (d) $\left(\sigma_{g} / \sigma_{g b}, J_{0, g} / J_{0, g b}\right)$. Note that stable propagation will always occur at either the grain or kink toughness. The ratio of strength and toughness controls the mechanism of propagation at each grain angle.

fracture are unstable and do not converge.

For each grain angle, the transition to intergranular fracture occurs at a particular normalized grain strength $\sigma_{g} / \sigma_{g b}$ and toughness $J_{0, g} / J_{0, g b}$. To remain consistent with the work of Parmigiani and Thouless (2006), we construct the curve of transition illustrated in Figure 10d. We also plot the corresponding single path solution (independent of $\sigma_{g} / \sigma_{g b}$ ) in Figure 10b to note the marked differences between modeling a single path or multiple paths of propagation. The intersection of the single path intergranular solution and the multiple path transgranular/intergranular solution occurs at a normalized grain strength of 
$\sigma_{g} / \sigma_{g b}=B=\sigma_{g} / \sigma_{g b}{ }^{I}$. The normalized grain strength of intersection $\sigma_{g} / \sigma_{g b}{ }^{I}$ is significant because it defines the role of grain strength. For $\sigma_{g} / \sigma_{g b}>\sigma_{g} / \sigma_{g b}{ }^{I}$, the grain strength aids the macroscopic toughness. The macroscopic resistance $J_{c}$ is greater than the input material resistance $J_{0, g b}$. For $\sigma_{g} / \sigma_{g b}<\sigma_{g} / \sigma_{g b}{ }^{I}$, the grain strength reduces the macroscopic toughness. The macroscopic resistance is actually less than the input material resistance.

\subsection{Penetration/deflection at $\theta=90^{\circ}$}

To illustrate role of grain strength and toughness, we plot curves of critical driving force and the resulting curve of intergranular fracture for a grain angle of $90^{\circ}$ in Figure 11. Over a narrow range of $J_{0, g} / J_{0, g b}$, the mode transitions from transgranular fracture (diagonal line) to intergranular fracture (horizontal line). We associate normalized grain strengths $\sigma_{g} / \sigma_{g b}$ of $2.7,3.0$, and 3.3 with "strong" grains. We note that grain strength enables deflection and yields an increased macroscopic toughness. If $\sigma_{g} / \sigma_{g b}=3.0$, we obtain a macroscopic toughness $J_{c}$ of $3.6 J_{0, g b}$ for an input grain toughness $J_{0, g}$ of $1.5 J_{0, g b} ; J_{c}$ is $2.4 J_{0, g}$.

In contrast, we identify normalized grain strengths of $\sigma_{g} / \sigma_{g b}$ of 2.0 and 2.1 with "weak" grains. Although $\sigma_{g} / \sigma_{g b}=2.1$ does eventually transition to intergranular fracture, substantial normalized grain toughness, $J_{0, g} / J_{0, g b}=6.12$, is required for transition. Transgranular cohesive zone formation effectively shields the intergranular cohesive zone. For a constant grain strength, an increased grain toughness requires a larger region at and near the peak traction in the transgranular cohesive zone. The body cannot provide the requisite, elevated stresses for the larger region and growth of the intergranular cohesive zone becomes favorable. Continued growth of the intergranular cohesive zone shields the grain and ensures intergranular propagation. The transition results in a loss in the macroscopic toughness. If $\sigma_{g} / \sigma_{g b}=2.1$, we obtain a macroscopic toughness $J_{c}$ of $3.6 J_{0, g b}$ for an input grain toughness $J_{0, g}$ of $6.1 J_{0, g b}$; $J_{c}$ is $0.59 J_{0, g}$. We also note that $\sigma_{g} / \sigma_{g b}$ of 2.3 and 2.5 border the single path solution. Within this narrow region, shielding is reduced and the body can sample both modes of propagation.

The intersection of the curves of critical driving force with the horizonal line of intergranular fracture in Figure 11a is used to construct the curve of transition plotted in Figure 11b. The intergranular solution is plotted for comparison. Curves of the intergranular and the transgranular/intergranular solutions intersect at $\sigma_{g} / \sigma_{g b}{ }^{I}=2.4$. For $\sigma_{g} / \sigma_{g b}>2.4$, the normalized grain strength aids the macroscopic toughness $\left(J_{c}>J_{0, g}\right)$. For $\sigma_{g} / \sigma_{g b}<2.4$, the normalized grain strength reduces the macroscopic toughness $\left(J_{c}<J_{0, g}\right)$. Although we note that the predicted transition results from the chosen grain boundary 

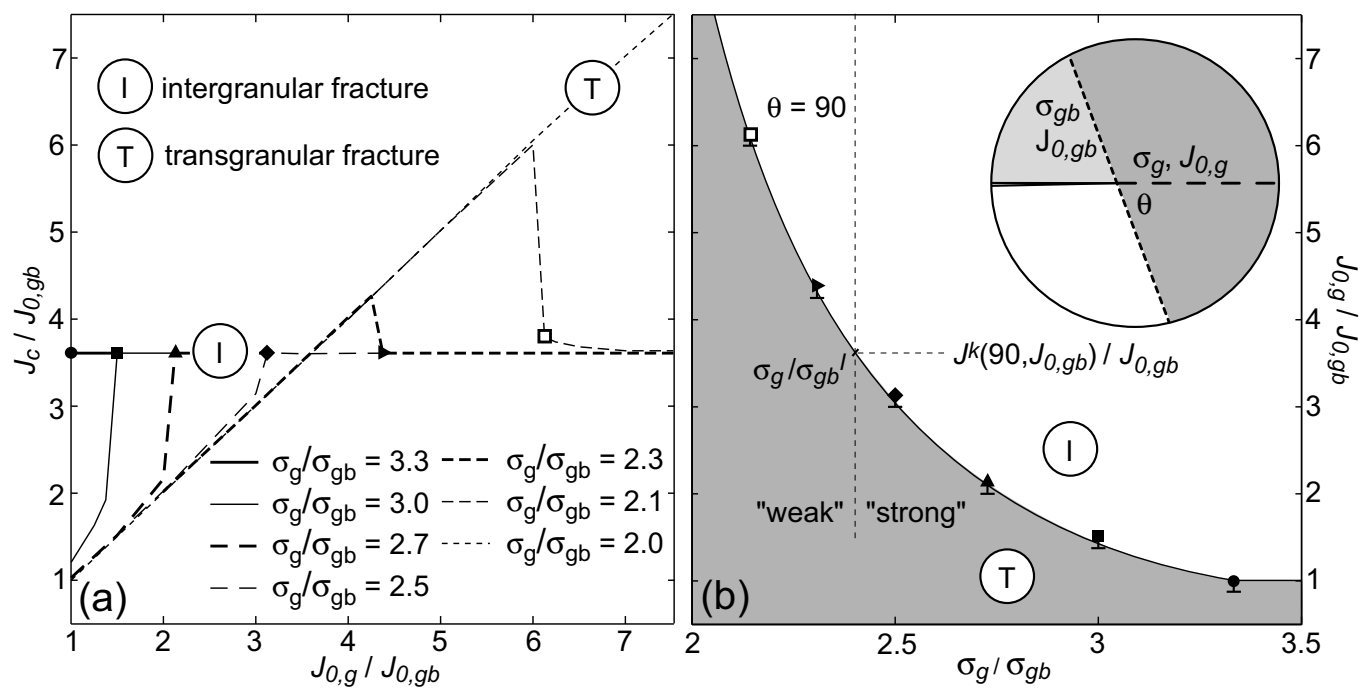

Fig. 11. Curves of (a) critical driving force and (b) transition to intergranular fracture for $\theta=90^{\circ}$. Stable propagation will always occur at either the grain $J_{0, g}$ or kink $J^{k}$ toughness. The normalized grain strength $\sigma_{g} / \sigma_{g b}$ and toughness $J_{0, g} / J_{0, g b}$ controls the mechanism of propagation. The intergranular and transgranular/intergranular solutions intersect at $\sigma_{g} / \sigma_{g b}{ }^{I}$.

properties, the significance of the transition is independent of properties and reflects a framework which incorporates both transgranular and intergranular fracture.

In addition to noting normalized trends, it is also important to consider the relevant length scale in the problem, the cohesive zone size $l_{c z}$. For $\theta=90^{\circ}$, the intergranular cohesive zone size for the grain boundary is on the order of $75 \mathrm{~nm}$. For "strong" grains, the transgranular cohesive zone size prior to the transition to intergranular fracture ranges between $8 \mathrm{~nm}\left(\sigma_{g} / \sigma_{g b}=3.0\right.$, $\left.J_{0, g} / J_{0, g b}=1.38\right)$ and $14 \mathrm{~nm}\left(\sigma_{g} / \sigma_{g b}=2.7, J_{0, g} / J_{0, g b}=2.00\right)$. Intermediate strengths of $\sigma_{g} / \sigma_{g b}=2.5, J_{0, g} / J_{0, g b}=3.00$ and $\sigma_{g} / \sigma_{g b}=2.3, J_{0, g} / J_{0, g b}=4.25$ bordering the energetic transition have cohesive zone sizes of $42 \mathrm{~nm}$ and $69 \mathrm{~nm}$, respectively. The cohesive zone size for "weak" grains varies between $114 \mathrm{~nm}$ $\left(\sigma_{g} / \sigma_{g b}=2.1, J_{0, g} / J_{0, g b}=6.00\right)$ and $178 \mathrm{~nm}\left(\sigma_{g} / \sigma_{g b}=2.0, J_{0, g} / J_{0, g b}=8.50\right)$. We note that for "weak" grains, the cohesive zone size approaches or exceeds the grain width, $150 \mathrm{~nm}$, in the prior study. Although we do not speculate the relevant range of cohesive zone sizes for this model system, we make the physical assertion that the transgranular cohesive zone does reflect a material process zone and should be smaller than microstructural dimensions. 


\subsection{Penetration/deflection for $\theta=82.5^{\circ}, 75^{\circ}, 67.5^{\circ}$, and $60^{\circ}$}

We also investigate the role of grain angle, as given by its inclination to the transgranular crack plane. Mirroring Figure 11a, Figure 12 illustrates curves of critical driving force for $\theta=82.5^{\circ}, 75^{\circ}$, and $67.5^{\circ}$. The $60^{\circ}$ configuration is not included in Figure 12 because all normalized grain strengths yield intergranular fracture. Again, we note that for stable propagation, the critical driving force is either the grain toughness $J_{0, g}$ or the kink toughness $J^{k}\left(J_{0, g b}, \theta\right)$. The normalized grain strength and grain toughness control the mode of propagation. As expected, the driving force required to kink the crack (horizontal line) decreases with $\theta$. Less obvious is the role of grain angle on the transition to intergranular fracture. For a constant normalized grain strength $\sigma_{g} / \sigma_{g b}$, the required normalized grain toughness $J_{0, g} / J_{0, g b}$ for deflection decreases markedly with decreasing $\theta$.

Perhaps a more meaningful interpretation can be derived under constant $J_{0, g} / J_{0, g b}$. As the grain angle $\theta$ decreases, the transition to intergranular fracture can occur at reduced normalized grain strength $\sigma_{g} / \sigma_{g b}$. Grains which would be considered to be of intermediate strength at $\theta=90^{\circ}$ easily deflect the crack at $\theta=75^{\circ}$. Under decreasing grain angle, no grains appear "weak"

- the transition to intergranular fracture occurs before $\sigma_{g} / \sigma_{g b}{ }^{I}$. Consequently, for the majority of normalized grain strengths, the macroscopic resistance $J_{c}$ is always greater than the grain resistance $J_{0, g}$.

Lower grain strengths are effective because of increased shielding and diminished loadings under reductions in grain angle. Detailed studies of intergranular fracture provided in Section 4 reveal that both the magnitude and extent of the principal stresses along the transgranular fracture plane reduce with decreasing grain angle. An increasing grain toughness $J_{0, g}$ requires a larger region at and near $\sigma_{g}$. Because reductions in grain angle decrease both the peak and region of elevated stress that the body can support for transgranular cohesive zone initiation, weaker and less tough grains are able to deflect the crack.

Curves of transition for decreasing grain angle are illustrated in Figure 13. Subsequential bisection of $J_{0, g}$ holding $\sigma_{g}$ and $\theta$ constant yields the transition to intergranular fracture within $J_{0, g b} / 8$. The single path, intergranular solutions are plotted for comparison. With decreasing grain angle, the cohesive curve of transition flattens and intersects the intergranular solution at reduced normalized grain strength. For $\theta=90^{\circ}, 82.7^{\circ}$, and $75^{\circ}, \sigma_{g} / \sigma_{g b}{ }^{I}$ is $2.40,2.37$, and 2.13 , respectively. We note that, for $\theta=67.5^{\circ}$, the intersection occurs outside the selected domain and that transgranular fracture did not occur for $\theta=60^{\circ}$. The current framework employing multiple paths of propagation reveals the importance of normalized grain strength under variation in grain angle. 


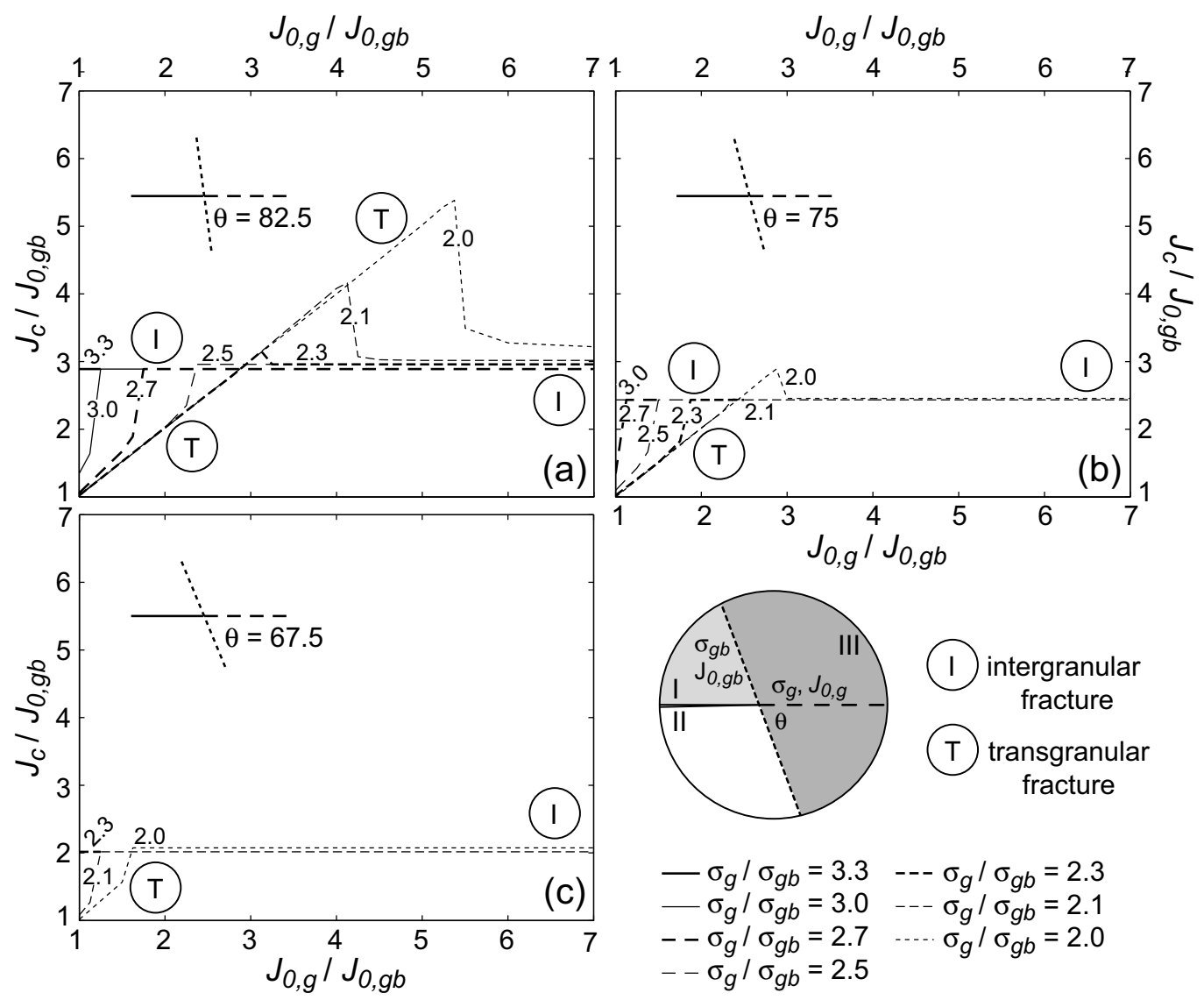

Fig. 12. Curves of critical driving force $J_{c}$ for varying grain boundary angle $\theta$. Transitions from transgranular fracture $J_{c}=J_{0, g}$ to intergranular fracture $J_{c}=J^{k}$ occur over very small changes in normalized grain toughness $J_{0, g} / J_{0, g b}$. The role of normalized grain strength $\sigma_{g} / \sigma_{g b}$ is magnified with decreasing grain angle.

\subsection{Impact of enabling transgranular and intergranular fracture}

To place the findings in perspective, we consider a few cases to illustrate that grain strength, toughness, and angle impact deflection. Specifically, we contrast the grain requirements for single (transgranular or intergranular) and multiple (transgranular and intergranular) path solutions. For infinitesimal deformations, the single path solution can be aligned with LEFM. At $90^{\circ}$, a single path solution in finite deformation for $\sigma_{g b}=E / 30$ requires $J_{0, g}=3.6 J_{0, g b}$ for deflection. However, if we enable both a transgranular and an intergranular path and assume the grain strength to be $\sigma_{g}=3.0 \sigma_{g b}$, the required grain toughness is only $1.5 J_{0, g b}$. If the grain strength is $3.3 \sigma_{g b}$, deflection occurs at $J_{0, g}=J_{0, g b}$. Note that although $J_{0, g}=J_{0, g b}$, the macroscopic toughness is $3.6 J_{0, g}$. Increases in the grain cohesive strength and grain shielding cause a change in mechanism, transgranular fracture $\rightarrow$ intergranular fracture, and result in macroscopic toughening. 


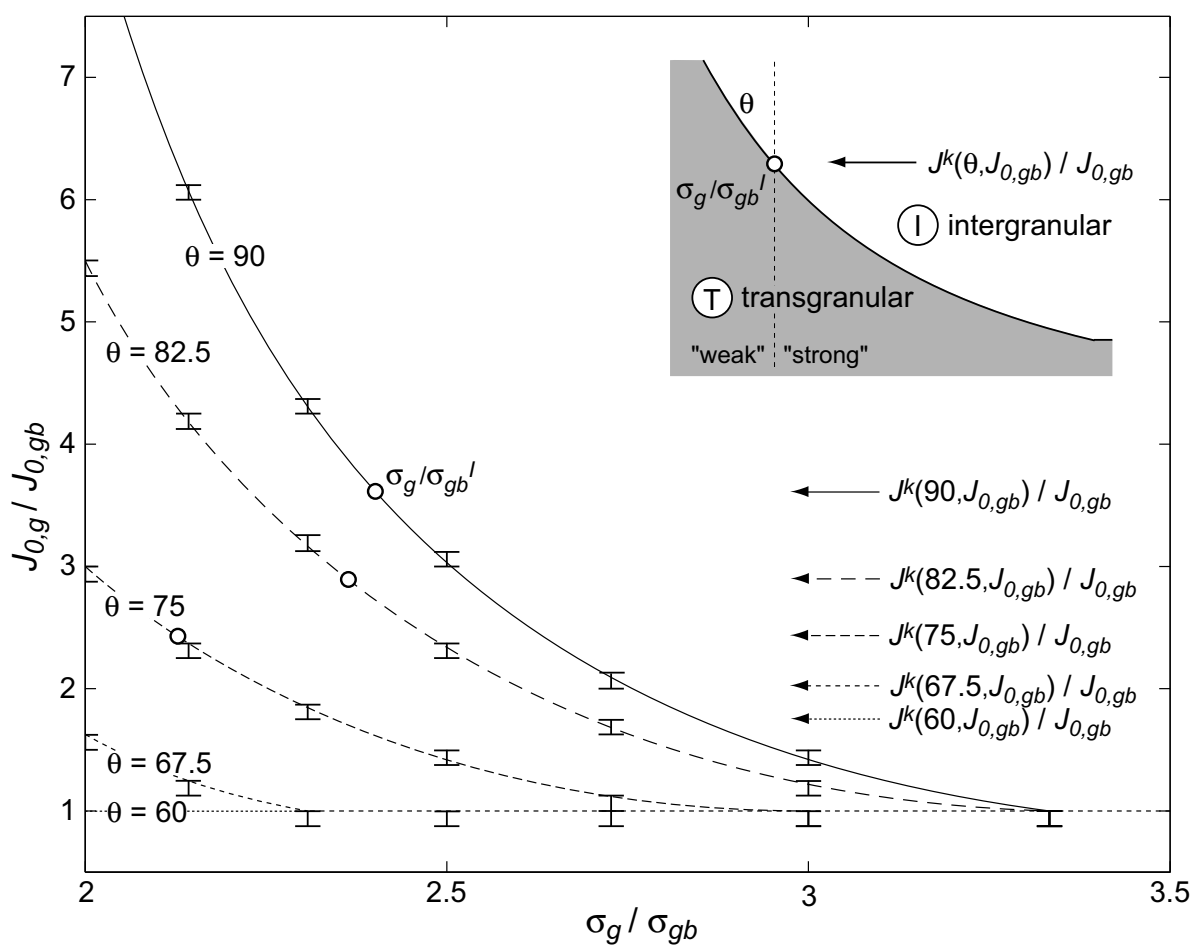

Fig. 13. Cross-sections of a surface of intergranular fracture at $\theta=90^{\circ}, 82.5^{\circ}, 75^{\circ}$, $67.5^{\circ}$, and $60^{\circ}$. The transition to intergranular fracture for each curve in Figure 12 represents a point on the $\left(\sigma_{g} / \sigma_{g b}, J_{0, g} / J_{0, g b}, \theta\right)$ surface. Subsequent bisection in $J_{0, g} / J_{0, g b}$ at constant $\sigma_{g} / \sigma_{g b}$ yields the transition to intergranular fracture within $J_{0, g b} / 8$. Curves of the single path intergranular solution $J^{k} / J_{0, g b}$ at each grain angle $\theta$ are plotted for comparison. The intersection $\sigma_{g} / \sigma_{g b} I$ of the single and multiple path solutions delineate "strong" and "weak" grains.

Consider now somewhat weaker grains and a decreasing grain angle. For $\sigma_{g}=2.5 \sigma_{g b}$, the required toughness for deflection at $\theta=90^{\circ}$ is slightly less than the single path solution, $J_{0, g}=3.1 J_{0, g b}$. For $82.5^{\circ}, 75^{\circ}$, and $67.5^{\circ}$, a single path solution requires a grain toughness of $2.9 J_{0, g b}, 2.4 J_{0, g b}$, and $2.0 J_{0, g b}$, respectively. The current approach considering multiple paths of propagation yields deflection for a grain toughness of $2.4 J_{0, g b}, 1.5 J_{0, g b}$, and $1.0 J_{0, g b}$ at grain angles of $82.5^{\circ}, 75^{\circ}$, and $67.5^{\circ}$, respectively. At decreased grain angles, cohesive zone initiation and growth along the grain boundary effectively shields the grain and less grain strength and toughness are required for deflection. Effects of both grain boundary angle and grain strength may shed light on the propensity of cracks in some material systems to penetrate at $90^{\circ}$ and deflect at decreased angles.

In addition to noting increases in the macroscopic toughness, we must also note normalized grain strengths which decrease the macroscopic toughness. We again consider the $90^{\circ}$ configuration and assume $\sigma_{g}=2.1 \sigma_{g b}$. To obtain a macroscopic toughness of $3.6 J_{0, g b}$, the grain toughness must be at least $6.1 J_{0, g b}$. For $\sigma_{g}=2.0 \sigma_{g b}$, the required toughness for deflection is 8.6 $J_{0, g b}$. We hesitate 
to report the required toughness for $\sigma_{g}=2.0 \sigma_{g b}$ because the cohesive zone size is on the order of the microstructure. Cohesive zone size aside, the trend is less than ideal. One would not continue to toughen the grain beyond the driving force required for deflection. For brittle materials, we seek to enable macroscopic toughness through local strength. Keeping the normalized grain

strength above $\sigma_{g} / \sigma_{g b}{ }^{I}$, the intersection of the intergranular and transgranular/intergranular solutions in Figure 13, we increase the macroscopic toughness through deflection.

\section{Application to structural ceramics}

Prior sections shed light on a cohesive approach to fracture and the required grain properties for maintaining an intergranular fracture path. Whereas an intergranular crack path is generally a sign of embrittlement in metallic materials, it is invariably a crucial event for the development of fracture toughness in ceramics; indeed, intergranular fracture and subsequent grain bridge formation provides the most potent mechanism for toughening most monolithic non-transforming structural ceramics. In our previous work (Foulk III et al., 2007), we focused on the evolution of grain bridging; specifically, toughening during the stall regime, prior to actual bridge formation, was shown to be substantial (see Figure 1) and to provide a basis for rapidly rising resistance curves. For grains oriented at $90^{\circ}$ and $67.5^{\circ}$ to the plane of the crack, the normalized macroscopic toughness $J_{c} / J_{0, g b}$ increases from 1 to 9 in one grain diameter. In the current work, we extend this approach to include transitions to transgranular fracture. Specifically, we have examined the conditions necessary to retain an intergranular crack path in order to preserve toughness. For consistency, we employ prior grain boundary properties and investigate a range of permissible grain properties. We remark that the model system is representative of structural ceramics and we seek to understand the ramifications of Section 4 and Section 5.

We contrast current findings with an energetic approach (He and Hutchinson, 1989) for the impingement of a crack at an interface. If we only consider a single path of propagation (penetration or deflection of the crack at the boundary), the cohesive zone approach and energetic approach will coincide for infinitesimal deformations. The cohesive strength and resulting cohesive zone size (provided it is small compared to the dimensions of the body) do not affect the driving force required for penetration or deflection. However, if multiple paths are explored simultaneously, both the cohesive strength and toughness affect the path of propagation (Parmigiani and Thouless, 2006). In addition to relieving the singularity, the current work illustrates that shielding is inherent in the cohesive zone approach to fracture. Prior to crack propagation, the intergranular and transgranular modes compete through cohesive 
zone formation. Cohesive zone growth in one mode shields the opposing mode from the stresses required for cohesive zone initiation. Curves of critical driving force and the resulting surfaces of intergranular fracture motivate thought experiments and provide a means for application.

Experimental observations have shown that the dominant mode of propagation for many brittle materials is intergranular fracture. Given the grain boundary properties and the intergranular path of propagation, one may attempt to infer the requisite grain properties for a crack impinging on an interface. Provided the transgranular path is aligned with the mode of loading, the required grain toughness can be calculated through a single path intergranular solution, $J_{0, g}=J^{k}\left(J_{0, g b}, \theta\right)$. The current work indicates that required toughness based on a single path is a special case of a more general framework considering both transgranular and intergranular fracture. For each grain angle $\theta$, the single and multiple path solutions coincide $J_{0, g}=J^{k}$ for a particular normalized grain strength $\sigma_{g} / \sigma_{g b}{ }^{I}$. If the grain is stronger $\sigma_{g} / \sigma_{g b}>\sigma_{g} / \sigma_{g b}{ }^{I}$, the crack deflects $J_{c}=J^{k}$ for $J_{0, g}<J^{k}$. If the grain is weaker $\sigma_{g} / \sigma_{g b}<\sigma_{g} / \sigma_{g b}{ }^{I}$, the crack still deflects $J_{c}=J^{k}$ but $J_{0, g}>J^{k}$. The trends noted in Figure 13 are clear: crack deflection is achieved through increasing grain strength.

Local shielding enables deviations from the single path solutions. Grains are considered "strong" if the body cannot provide the requisite tractions for transgranular cohesive zone initiation. Intergranular cohesive zones will initiate, grow, and further shield the transgranular cohesive zone. Grains are considered "weak" if the body can provide the requisite tractions for transgranular cohesive zone initiation and growth. The transgranular cohesive zones will initiate, grow and further shield the intergranular cohesive zones. In the transition to intergranular fracture, the grain toughness does not impact the transgranular cohesive zone size $l_{c z}$ at propagation. Rather, the normalized grain toughness $J_{0, g} / J_{0, g b}$ controls the size of the region in the transgranular cohesive zone at and near the peak strength. For a given normalized grain strength $\sigma_{g} / \sigma_{g b}$, Figures 11a and 12 illustrate that the transition to intergranular fracture is abrupt and sensitive to the ratio of grain to grain-boundary toughness, $J_{0, g} / J_{0, g b}$. For "strong" $\sigma_{g} / \sigma_{g b}>\sigma_{g} / \sigma_{g b}{ }^{I}$ grains, small increases in $J_{0, g} / J_{0, g b}$ can result in dramatic increases in the normalized macroscopic toughness $J_{c} / J_{0, g b}$. We also note that if the grains are "weak" $\sigma_{g} / \sigma_{g b}<\sigma_{g} / \sigma_{g b}{ }^{I}$, small increases in $J_{0, g} / J_{0, g b}$ can result in dramatic decreases in the normalized macroscopic toughness $J_{c} / J_{0, g b}$. Decreases in $\theta$ from $90^{\circ}$ promote intergranular cohesive zone formation and shield the grain. Consequently, at $\theta=75^{\circ}$, almost all normalized grain strengths $\sigma_{g} / \sigma_{g b}=2.1 \rightarrow 3.3$ appear "strong" and necessarily deviate from the single path solution.

Additional simulations not presented in this work were conducted in the stall regime prior to crack reinitiation. Those simulations confirm that substantial grain strength $\sigma_{g}=4.0 \sigma_{g b}$ with moderate grain toughness $J_{0, g}=2.6 J_{0, g b}$ main- 
tains an intergranular path and yields a rapid rise in the resistance. Curves of critical driving force delineating transgranular fracture, intergranular reinitiation, and the unstable transition from transgranular to intergranular fracture were constructed for particular crack configurations. The resulting curves of reinitiation, similar to the curves of intergranular fracture noted in Figure 13, were also generated but not included to focus on the fundamental issues presented in Section 4 and Section 5.

\section{Conclusions}

Maintaining an intergranular fracture path is a requirement for the generation of the most potent toughening mechanism in (non-transforming) monolithic ceramics, that of grain bridging. Although prior work focused on bridge formation and the elevated toughening achieved during the stall regime, the path of propagation was constrained to the grain boundaries. In this work, we enable intergranular and transgranular fracture and specify the requisite grain properties to maintain an intergranular path. Employing a cohesive zone approach to fracture, we thoroughly investigate particular crack configurations for a constant grain boundary strength and toughness under a variance in grain strength, toughness, and angle. Targeted studies of the competition between intergranular and transgranular fracture for relevant crack configurations not only yield the grain properties necessary to preserve macroscopic toughness but also provide a baseline for numerical methods addressing generalized fracture.

Through targeted studies of a crack penetrating or deflecting at an interface, we find that:

(1) If the interaction between transgranular and intergranular cohesive zones is stable, propagation will occur at either the grain or kink toughness. The mode of propagation, however, is a function of the normalized grain strength, normalized grain toughness, and grain angle.

(2) Curves of critical driving force at constant normalized grain strength trace the abrupt and unstable transitions from transgranular to intergranular fracture.

(3) An increase in the normalized grain toughness at constant normalized grain strength requires that a larger region of the transgranular cohesive zone be at and near the grain strength. Because the body cannot provide the requisite field for the increasing region, the mode of propagation rapidly transitions from transgranular to intergranular fracture.

(4) There exists a normalized grain strength at each grain angle, the normalized grain strength of intersection, that yields the single path solution considering either transgranular or intergranular fracture. For infinites- 
imal deformations, the normalized grain strength of intersection yields the energetic solution given by LEFM.

(5) Grains stronger than the normalized grain strength of intersection encourage intergranular cohesive zone formation, enable grain shielding, and increase the macroscopic toughness. For $\theta=90^{\circ}, \sigma_{g} / \sigma_{g b}=3.3, J_{c}$ is $3.6 J_{0, g}$.

(6) Grains weaker than the normalized grain strength of intersection encourage transgranular cohesive zone formation, enable grain boundary shielding, and decrease the macroscopic toughness. For $\theta=90^{\circ}, \sigma_{g} / \sigma_{g b}=2.1$, $J_{c}$ is $0.6 J_{0, g}$.

(7) Decreases in grain angle promote intergranular cohesive zone formation and shield the grain. Increased shielding from decrements in grain angle lessen the normalized grain strength and toughness necessary to maintain an intergranular fracture path.

(8) The competition between multiple paths of propagation yields a surface of intergranular fracture in normalized grain strength, normalized grain toughness, and grain angle. The intersection of the multiple path and single path solutions yields the normalized grain strength of intersection.

In this work, we highlight the role of normalized grain strength, normalized grain toughness, and grain angle in promoting intergranular fracture. Both forms of bridging, due to interlocking grains and uncracked ligaments, which are essential for macroscopic toughness, require this mode of fracture. An intergranular path including crack deflection and crack stalling prior to bridge formation generates a rapid rise in the resistance curve and yields macroscopic strength at realistic flaw sizes. Current findings illustrate that the experimentally observed intergranular path, bridging mechanisms, and rapid rise in the resistance can be achieved through substantial grain strength and moderate grain toughness.

\section{Acknowledgements}

We are indebted to our late colleague, Dr. Rowland Cannon, for his invaluable discussion and only hope that this work is of sufficient complexity to honor his memory. J.W.F. is grateful for the support of Sandia National Laboratories, operated by Sandia Corporation, a Lockheed Martin Company, for the United States Department of Energy under contract DE-AC04-94AL85000. R.O.R. acknowledges the support provided by the Director, Office of Science, Office

of Basic Energy Sciences, Division of Materials Sciences and Engineering of the U.S. Department of Energy under Contract No. DE-AC02-05CH11231. We also thank Drs. B.L. Boyce and E.D. Reedy, Jr. for their helpful comments on the manuscript. 


\section{References}

Arata, J.J.M., Kumar, K.S., Curtin, W.A., Needleman, A., 2001. Crack growth in lamellar titanium aluminide. International Journal of Fracture 111, 163189.

Arata, J.J.M., Kumar, K.S., Curtin, W.A., Needleman, A., 2002. Crack growth across colony boundaries in binary lamellar TiAl. Materials Science and Engineering A329-331, 532-537.

Ballarini, R., Kahn, H., Tayebi, N., Heuer, A.H., 2001. Effects of microstructure on the strength and fracture toughness of polysilicon: a wafer level testing approach. In: Muhlstein, C.L., Brown, S.B. (Eds.), Mechanical properties of structural films, STP 1413. American Society for Testing and Materials, pp. 37-51.

Becher, P.F., Hsueh, C.-H., Alexander, K.B., Sun, E.Y., 1996. Influence of reinforcement content and diameter on the $\mathrm{R}$-curve response in $\mathrm{SiC}$-whiskerreinforced alumina. Journal of the American Ceramic Society 79, 298-304.

Becher, P.F., Sun, E.Y., Plucknett, K.P., Alexander, K.B., Hsueh, C.-H., Lin, H.-T., Waters, S.B., Westmoreland, C.G., 1998. Microstructural design of silicon nitride with improved fracture toughness: I, effect of grain size and shape. Journal of the American Ceramic Society 81, 2821-2830.

Chan, K.S., Onstott, J., Kumar, K.S., 2000. The fracture resistance of a binary TiAl alloy. Metallurgical and Materials Transactions A31, 71-80.

Chantikul, P., Bennison, S.J., Lawn, B.R., 1990. Role of grain size in the strength and R-curve properties of alumina. The Journal of the American Ceramic Society 73, 2419-2427.

Cotterell, B., Rice, J.R., 1980. Slightly curved or kinked cracks. International Journal of Fracture 16, 155-169.

Eshelby, J.D., 1951. The force on an elastic singularity. Philosophical Transactions of the Royal Society of London A244, 87-112.

Espinosa, H.D., Zavattieri, P.D., 2003a. A grain level model for the study of failure initiation and evolution in polycrystalline brittle materials. part I: theory and numerical implementation. Mechanics of Materials 35, 323-364.

Espinosa, H.D., Zavattieri, P.D., 2003b. A grain level model for the study of failure initiation and evolution in polycrystalline brittle materials. part II: numerical examples. Mechanics of Materials 35, 365-394.

Faber, K.T., Evans, A.G., 1983. Crack deflection processes. I. theory. Acta Metallurgica 31, 577-584.

Foulk III, J.W., Cannon, R.M., Johnson, G.C., Klein, P.A., Ritchie, R.O., 2007. A micromechanical basis for partitioning the evolution of grain bridging in brittle materials. Journal of the Mechanics and Physics of Solids 55, 719-743.

Gilbert, C.J., Cao, J.J., MoberlyChan, W.J., De Jonghe, L.C., Ritchie, R.O., 1996. Cyclic fatigue and resistance-curve behavior of an in situ toughened silicon carbide with Al-B-C additions. Acta Materialia 44, 3199-3214.

He, M.-Y., Hutchinson, J.W., 1989. Kinking of a crack out of an interface. 
Journal of Applied Mechanics 56, 270-278.

Hutchinson, J.W., Suo, Z., 1991. Mixed mode cracking in layered materials. Advances in Applied Mechanics 29, 63-187.

Klein, P.A., Foulk, J.W., Chen, E.P., Wimmer, S.A., Gao, H.J., 2001. Physicsbased modeling of brittle fracture: cohesive formulations and the application of meshfree methods. Theoretical and Applied Fracture Mechanics 37, 99166.

Knehans, R., Steinbrech, R., 1982. Memory effect of crack resistance during slow crack growth in notched $\mathrm{Al}_{2} \mathrm{O}_{3}$. Journal of Materials Science Letters $1,327-329$.

Kovalev, S., Miyajima, T., Yamauchi, Y., Sakai, M., 2000. Numerical evaluation of toughening by crack-face grain interlocking in self-reinforced ceramics. Journal of the American Ceramic Society 83, 817-824.

Kruzic, J.J., Cannon, R.M., Ager III, J.W., Ritchie, R.O., 2005. Fatigue threshold R-curves for predicting reliability of ceramics under cyclic loading. Acta Materialia 53, 2595-2605.

Maiti, S., Rangaswamy, K., Geubelle, P.H., 2005. Mesoscale analysis of dynamic fragmentation of ceramics under tension. Acta Materialia 53, 823834.

Needleman, A., 1987. A continuum model for void nucleation by inclusion debonding. Journal of Applied Mechanics 54, 525-531.

Needleman, A., 1990. An analysis of tensile decohesion along an interface. Journal of the Mechanics and Physics of Solids 38, 289-324.

Parmigiani, J.P., Thouless, M.D., 2006. The roles of toughness and cohesive strength on crack deflection at interfaces. Journal of the Mechanics and Physics of Solids 54, 266-287.

Qiao, Y., Argon, A.S., 2003a. Cleavage crack-growth-resitance of grain boundaries in polycrystalline Fe-2\%Si alloy: experiments and modeling. Mechanics of Materials 35, 129-154.

Qiao, Y., Argon, A.S., 2003b. Cleavage cracking resistance of high angle grain boundaries in Fe-3\%Si alloy: experiments and modeling. Mechanics of Materials $35,313-331$.

Rice, J.R., 1968. A path independent integral and the approximate analysis of stress concentration by notches and cracks. Journal of Applied Mechanics 35, 379-386.

Ritchie, R.O., Gilbert, C.J., McNaney, J.M., 2000. Mechanics and mechanisms of fatigue damage and crack growth in advanced materials. International Journal of Solids and Structures 73, 311-329.

Simo, J.C., Hughes, T.J.R, 1997. Computational Inelasticity. Springer-Verlag, New York.

Simo, J.C., Taylor, R.L., Pister, K.S., 1985. Variational and projection methods for the volume constraint in finite deformation elastoplasticity. Computer Methods in Applied Mechanics and Engineering 51, 177-208.

Swanson, P.L., Fairbanks, C.J., Lawn, B.R., Mai, Y.-W., Hockey, B.J., 1987. Crack-interface grain bridging as a fracture resistance mechanicsm in ce- 
ramics: I, experimental study on alumina. Journal of the American Ceramic Society 70, 279-289.

Tvergaard, V., Hutchinson, J.W., 1990. Effect of fiber debonding in a whiskerreinforced metal. Materials Science and Engineering A125, 203-213.

Tvergaard, V., Hutchinson, J.W., 1992. The relation between crack growth resistance and fracture process parameters in elastic-plastic solids. Journal of the Mechanics and Physics of Solids 40, 1377-1397.

Tvergaard, V., Hutchinson, J.W., 1993. The influence of plasticity on mixed mode interface toughness. Journal of the Mechanics and Physics of Solids 41, 1119-1135.

Xu, H.H.K., Wei, L., Padture, N.P., Lawn, B.R., Yeckley, R.L., 1995. Effect of microstructural coarsening on short-crack toughness properties of silicon nitride. Journal of Materials Science 30, 869-878.

Yuan, R., Kruzic, J.J., Zhang, X.F., De Jonghe, L.C, Ritchie, R.O., 2003. Ambient to high-temperature fracture toughness and cyclic fatigue behavior in Al-containing silicon carbide ceramics. Acta Materialia 51, 6477-6491.

Zavattieri, P.D., Raghuram, P.V., Espinosa, H.D., 2001. A computational model of ceramic microstructures subjected to multi-axial dynamic loading. Journal of the Mechanics and Physics of Solids 49, 27-68. 